\title{
LUT
}

University

\section{A novel haptic interface and universal control strategy for International Thermonuclear Experimental Reactor (ITER) welding/machining assembly robot}

Roozbahani Hamid, Handroos Heikki

This is a Final draft version of a publication

published by Elsevier

in Robotics and Computer-Integrated Manufacturing

DOI: $\quad 10.1016 /$ j.rcim.2018.12.011

Copyright of the original publication: (c) 2018 Elsevier Ltd.

Please cite the publication as follows:

Roozbahani, H., Handroos, H. (2019). A novel haptic interface and universal control strategy for International Thermonuclear Experimental Reactor (ITER) welding/machining assembly robot. Robotics and Computer-Integrated Manufacturing, vol. 57, pp. 255-270. DOI: 10.1016/j. rcim.2018.12.011

This is a parallel published version of an original publication. This version can differ from the original published article. 


\title{
A Novel Haptic Interface and Universal Control Strategy for International Thermonuclear Experimental Reactor (ITER) Welding/Machining Assembly Robot
}

\author{
${ }^{1}$ Hamid Roozbahani, ${ }^{2}$ Heikki Handroos \\ ${ }^{1,3}$ Department of Mechanical Engineering, Lappeenranta University of Technology \\ P.O.Box 20, Lappeenranta, FI-53850,Finland, Corresponding Author: hamid.roozbahani@lut.fi
}

\begin{abstract}
This paper proposes a universal control strategy and novel haptic interface for the International Thermonuclear Experimental Reactor (ITER) welding/machining robot. The developed method implements a reliable position control and sense between the human and the robot as well as between the robot and the task environment in which provides highly accurate position control based on joystick inputs with real-time haptic capabilities. The proposed control structure has the characteristics of a universal technique independent of the actual control algorithm and it can be applied with other suitable control methods as a real-time control strategy. One of the contributions of this paper is that the proposed control method combines a directed random search method and real-time simulation to develop an intelligent controller in which each generation of parameters is tested on-line by the real-time simulator before being applied to the real process. The controller was evaluated on a hydraulic position servo system where the simulator of the hydraulic system was built based on Markov chain Monte Carlo (MCMC) method. A Particle Swarm Optimization (PSO) algorithm combined with the foraging behavior of E. coli bacteria was utilized as the directed random search engine. PSO is influenced by the simulation of social behavior in which each individual agent of the possible solution population, benefits from its history and its interactions with other agents within the population. This sharing of knowledge helps facilitate faster convergence to an optimal solution. From the other side, the effect of Bacterial Foraging Optimization (BFO) of E coli is that the agent is making a decision to which position to move. The agent does this with attention to the previous data stored in the memory as the best past position. These selection behaviors of particles help to avoid poor foraging and improve foraging strategies. In conclusion, the proposed control and haptic strategy allows the operator to be kinetically plugged into the work environment and simultaneously provides force feedback sense with high controllability without neglecting the system dynamics.
\end{abstract}

Keywords - International Thermonuclear Experimental Reactor (ITER), Haptic, Intelligent Control, Teleoperated hydraulic manipulator, Real-time Simulation, Particle Swarm Optimization (PSO), Bacterial Foraging Optimization (BFO) of E coli

\section{Introduction}

PC-based controllers have recently become quite powerful. Because of this, it is possible to utilize more and more complex models and algorithms in real-time control. The classical approach has been using a linear observer (Luenberg, Kalman) to approximate the missing sensor signals.

Numerical optimization methods become significantly powerful for controller parameter tuning by using new computational devices [1]. Directed random search methods such as Genetic Algorithm (GE) and Differential Evolution (DE) have been widely applied in the field of machine learning and control engineering [2,3]. They have been extremely capable in finding global optimums in the presence of nonlinearities, and they are able to effectively solve discrete optimization problems. However, in machine learning, those algorithms have serious drawbacks such as unstable generations and slow convergence speed. Their applications in tuning controllers and the optimization of controller structures have widely been discussed in the literature. In addition, their practical applications are limited because of the damage threats from their instability, which is not acceptable in most cases $[4,5]$.

Most of the neural network based controllers proposed in the field of robotics use feed-forward type of neural networks and they use back-propagation algorithms in learning. The back-propagation is a gradient-based optimization algorithm for updating the weights and biases of the network during each learning cycle. It has bad stability in the presence of discontinuity, high stiffness and local minima that restricts the use of neural control in the major applications in practice [6].

Previously completed and on-going research projects have shown that it is possible to simulate complex dynamic models for various mechatronic machines in real-time. Several simulation models for electric, hydraulic and pneumatic servo systems as well as various types of serial and parallel manipulators have recently been postulated [7]. Instead of approximating feedback signals using a linear observer as happens using GE or DE, this study uses a nonlinear real-time simulator in parallel with the real system, which a real-time simulator tests each generation of control parameters before applying into the real process $[8,9]$.

This study proposes a novel method, which combines directed random search and real-time simulation for developing intelligent controller for tele-operated servo systems. This control method provides a reliable haptic sense and control capabilities in case of contact with the environment. The technique enhances the manipulator performance under various environmental circumstances and regardless of disturbances for the tele-operated inputs. The most important advantage of the proposed method is its online real-time characteristic, which provides the best available control parameters for the system.

Applying the directed random search methods has positive effects on the controller structure in which the control parameters can be optimized to achieve good control properties. The key problem that restricts the use of directed random search methods is the generation of control parameters that cause instability during optimization. To overcome this problem, in this research, each generation of control parameters is tested on-line by a real-time simulator before application in the real process. The reason of developing such control strategy is that, a traditional linear controller, generally, provides an acceptable performance during the most of the operating range. Nevertheless, it cannot protect this acceptable performance during the whole operation; especially when external disturbances and 
environmental interactions are also involved in the operation [10]. In this research, the controller-tuning algorithm is based on Particle Swarm Optimization which is combined with the foraging behavior of E coli bacteria. The PSO algorithm could lead to local solutions and the E coli algorithm may lead to a delay in reaching a global solution. However, the combination of both algorithms could lead to better optimization [11,12]. During optimization, the reference input and the simulated output are used to calculate the cost function for the particle swarm optimization algorithm. This leads to optimum control parameters and avoids bad combinations, which normally appear during optimization with the directed random search method.

Swarming strategies of bird flocking and fish schooling are used in the Particle Swarm Optimization introduced by Eberhart and Kennedy in 1995 [13]. PSO has several advantages in comparison to neural-based methods such as genetic algorithms (GA). PSO relies on a memory-based progression, in which the previous solutions are remembered and continually improved upon until convergence is reached $[14,15]$. In comparison, genetic algorithms suffer from premature convergence since they rely on genetic operators that allow weak solutions to contribute to the composition of future candidate solutions. Traditional tuning methods also require further fine-tuning to improve control performance [16]. On the one hand, PSO is influenced by the simulation of social behavior rather than survival of the fittest as in the GA [17]. On the other hand, the use of simple mathematical operators allows faster computational time and makes the algorithm suitable for determining tuning parameters under high-speed dynamical conditions for processes that lend themselves to tuning of this nature, such as flow and pressure control. Tuning parameters obtained with PSO are consistent over a number of tuning sessions. This does not apply to the GA-based tuning method [18].

The basic idea of this research is to use a real-time simulator in parallel with the real process and to develop an intelligent switching method that selects either a linear or an intelligent controller, according to which of these currently provides more accurate system behavior. In this control strategy, the intelligent controller controls the real-time simulator and optimizes with the simulation model.

After improvement, because of the simulated response, the improved controller is switched to control the real system. The switching criterion is the comparison between the current simulation model output and the real system output. From the results presented in the thesis, PSO tuning yielded improved responses and can be applied to different process models encountered in the process control industry.

The main task in ITER project is to design and develop a mobile robot to conduct welding and milling inside the ITER chamber. The necessity of having such a robot is the radioactive environment inside the ITER chamber after the first fusion. As a part of the development of the main robot, it was necessary to design and develop a proper position control and haptic system for the robot which has $6+4$ degrees of freedom. Therefore it was decided to develop the controller and first apply it to a 1DOF system to make sure of reliability of the developed control and haptic algorithm and then the controller has applied to the main robot. The application of the controller to the main robot is the subject of upcoming new publications in near future.

The main reason behind the need for such a control system for the ITER robot is that the accuracy of welding and milling inside the ITER chamber is a very important factor and there is no room for any errors. Therefore it was important to develop a type of controller that first applies the control parameters to an accurate simulation of the robot and then in case the results are good then to apply the control parameters to the main robot. In addition, the main reason behind the need for haptic and force feedback was that the operator does not have direct access to the robot or the environment that the robot is working in. The access is mostly from cameras and also a simulation animation of the ITER chamber.

\subsection{International Thermonuclear Experimental Reactor (ITER)}

The international thermonuclear experimental reactor (ITER) is a joint international research and development project that aims to demonstrate the scientific and technical feasibility of fusion power. The reactor is based on the Tokamak principle concept, in which the $150 \mathrm{M}^{\mathrm{oC}}$ plasma is enclosed inside a vacuum vessel by strong magnetic fields created by super conductor coil magnets. The vacuum vessel is made of stainless steel, and it consists of nine sectors welded together $[39,40]$. (See Figure 1)

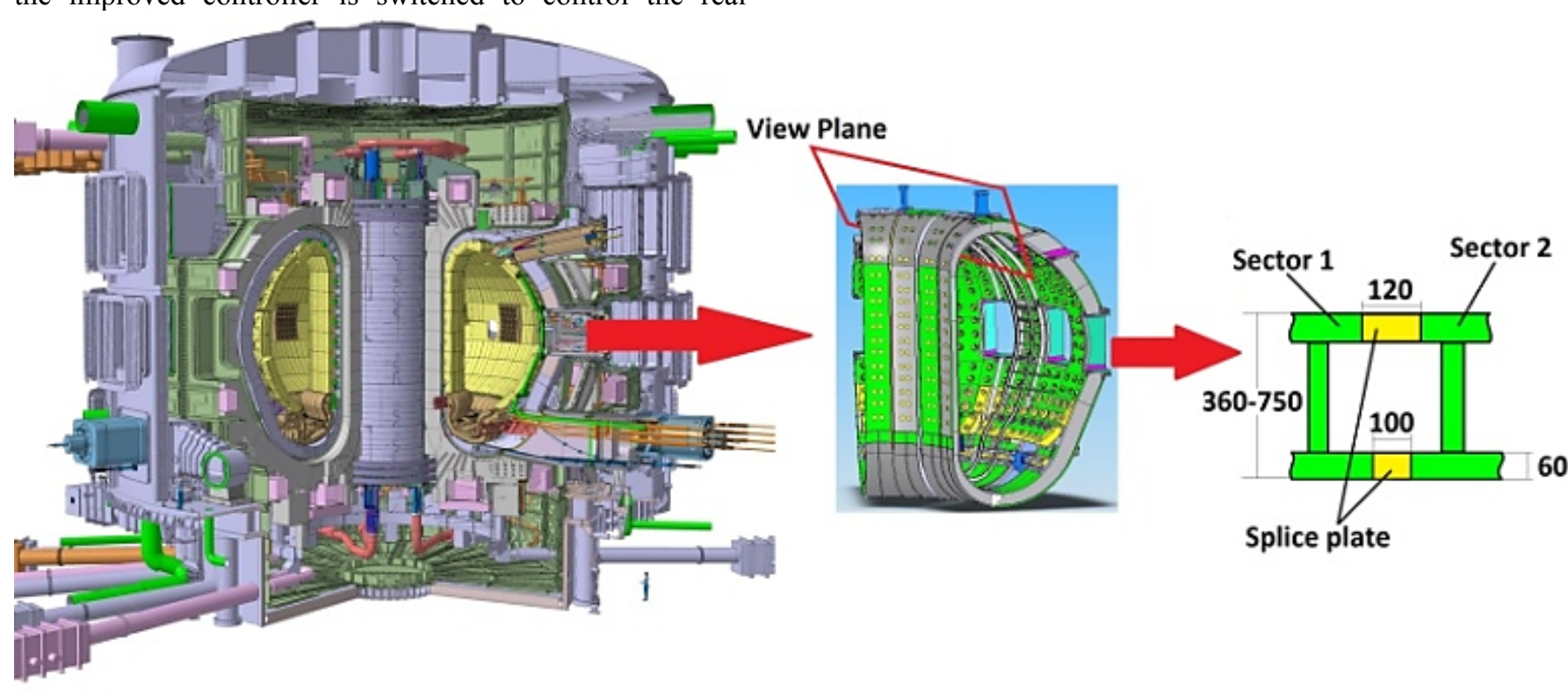

Fig. 1. ITER and VV sectors to be welded [1] 
Each sector is about $11 \mathrm{~m}$ high and $6 \mathrm{~m}$ wide. The vacuum vessel requires a very tight absolute tolerance $( \pm 5$ $\mathrm{mm})$ to achieve sufficiently accurate magnetic focus. The vacuum-vessel sectors have a double-shell structure using formed $60 \mathrm{~mm}$ thick stainless steel $(316 \mathrm{~L})$ plates. To compensate the effects of manufacturing errors on the overall tolerance of the vacuum vessel, tailor made splice plates are inserted between the sectors to be joined by leaktight welds [2].

In addition to the first assembly, the sectors have to be removed later for repair and re-assembly. A high quality narrow gap welding process is required to attain leak-tight seams and ensure minimal deformations during the welding.

In addition to welding, various other tasks have to be completed during assembly by a specially designed robotic system. These tasks range from machining defect welds to handling $150 \mathrm{~kg}$ splice plates and in-wall shield blocks [39, 40].

All the tasks have to be carried out from inside the vessel because the supra magnets are already assembled around the sectors before joining. A major problem with the ITER robot is difficulty accessing the robot when it operates inside the ITER Vacuum Vessel (VV), especially after firing of the first plasma, which will make the vessel radioactive. Commercially available industrial robots are unsuitable for such operations because they are incapable of handling sufficiently large payloads in such small spaces and require floor mounting. Their stiffness is also insufficient for machining [1].

\subsection{The Assembly robot}

Due to un availability of a reliable commercial robot for ITER project vessel assembly, Lappeenranta University of Technology (LUT) have been developed a tailor-made parallel robotic solution for carrying out various tasks during assembly of the ITER vacuum vessel. The robot presented in Figure 2 has many superior properties compared to commercially available industrial robots, as described in [3] and [4].

The robot's parallel kinematic hydraulic Stewartplatform provides high stiffness and its additional electrically driven serial degrees of freedom provides an extended work space for the end effector. The robot offers not only a device but also a methodology for assembling and repairing the vacuum vessel. The end effector of the robot has to pass through the inner wall splice plate opening to reach the outer wall. The assembly and repairing processes have to be carried out from inside the vacuum vessel (See Figure 3.) [1].

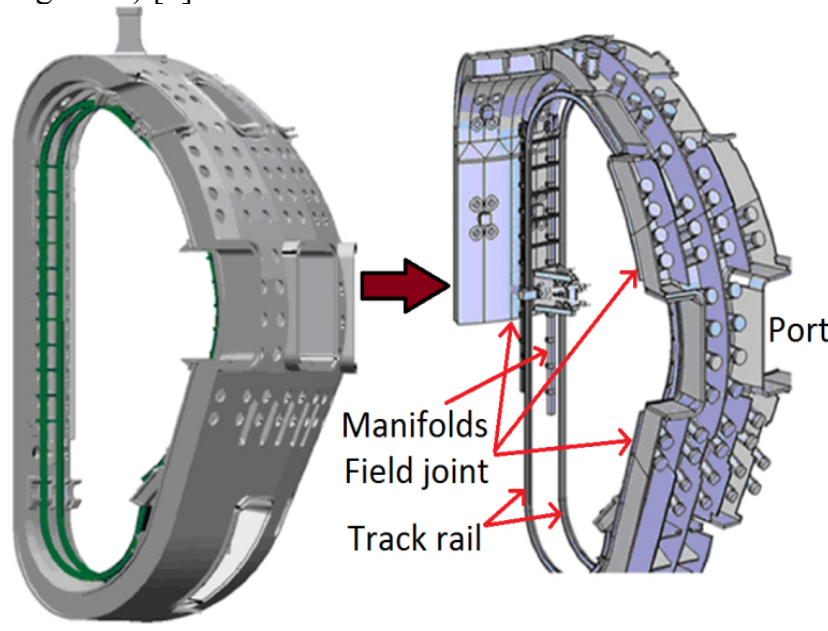

Fig. 3. The track rail mounted inside VV and robot on the track

To reach the outer wall, the robot's end-effector has to pass through the inner wall splice plate opening. As can be seen from Figure 3, the assembly and repairing processes have to be carried out from inside the vacuum vessel [2]-[4]

\section{Control algorithm}

In this study, a non-linear real-time simulator in parallel with the real system is used. This approach is a novel method that combines directed random search and real-time simulation. The method has the characteristics of a universal technique independent of the actual control algorithm and it can be applied with other suitable control methods as a realtime control strategy.

The most important advantage of the proposed method is the online real-time characteristic, which provides the best available control parameters for the robot. Figure 4 illustrates the global scheme of the proposed control strategy, in which $Y_{m}$ is the real system output and $Y_{s}$ is the simulator output.

In this control method, the real-time simulator is equipped with the intelligent controller and the real system is equipped with a linear controller. Both systems are fed with the same input.
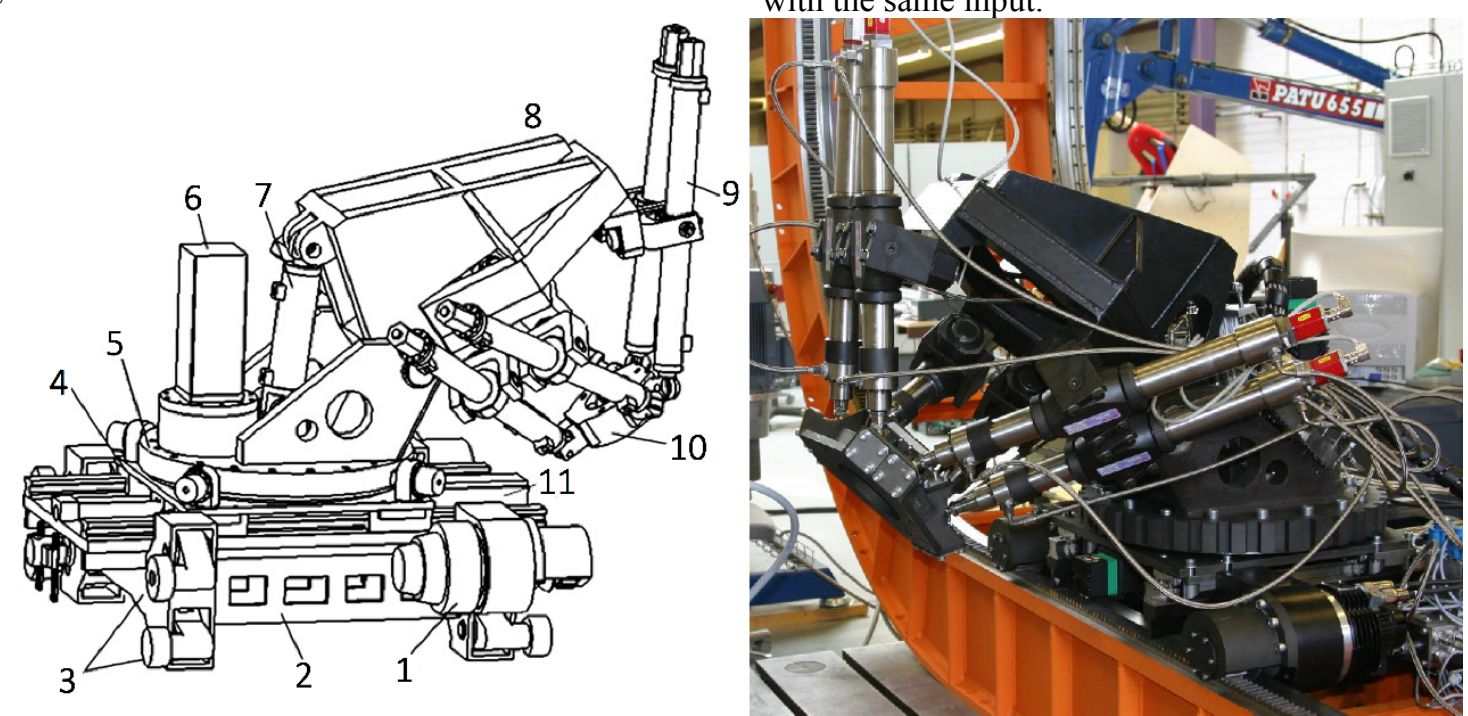

Fig. 2. ITER Parallel robot. Robot parts: 1.Drive motor 2.Carriage frame 3.Compensate mechanism 4.Linear table 5.Rotation table 6.Rotation drive unit 7.Tip drive cylinder 8.Hexa-WH frame 9.Hexa-WH drive cylinder 10.End effector 11.Linear drive motor 


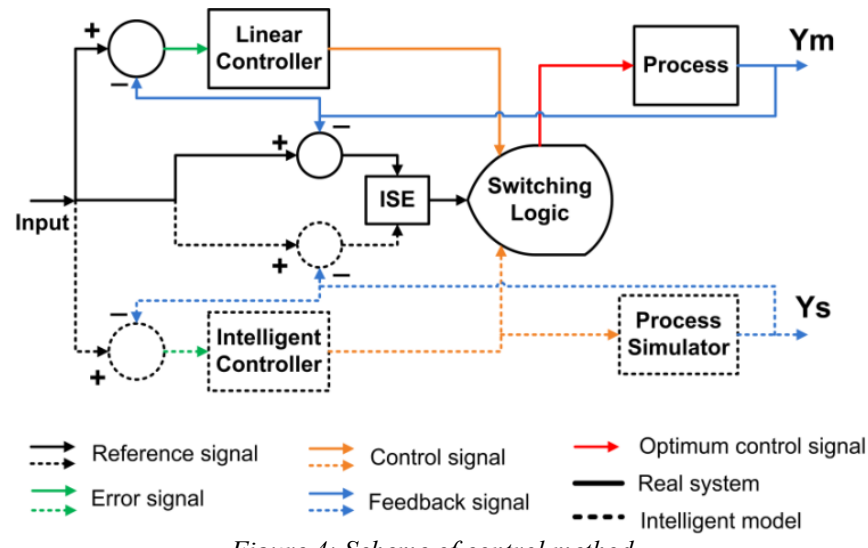

Figure 4: Scheme of control method

The main role of the intelligent controller is to search for optimal parameters for the real system; it does not have the authority to control the real system directly but it controls the real-time simulator throughout. Whenever the controller finds a set of control parameters with smaller cost function value, this set of parameters is used to control the real system via an intelligent switch. The switching criterion is a comparison between the current simulated control output and real system output Integral Square Error (ISE) cost function. The transition is smoothened to avoid transmitting disturbances into the real system. The real system continues working with the new set of control parameters until a better set appears. Figure 5 presents the structure of the intelligent controller, simulator and intelligent switch in connection with the real system. It should be noticed that there are different types of cost functions to find the best control values in this type of optimization. With attention to the high accuracy and satisfactory results, which Integral of the Squared Error (ISE) provided during the design of switch, it has been chosen as the cost function in this optimization project. ISE is defined as:

Min $: e 2=\int_{0}^{T} e^{2} \mathrm{dt}$

where $\mathrm{T}$ is the present time step and e is the system error. If the error in any time step becomes large, then the integral cumulative effect may become significant. In order to avoid this problem, the algorithm utilizes a forgetting factor, i.e., after calculating the cost function for time duration of $\mathrm{T}$, the cost function value is set back to zero.

\subsection{Particle Swarm Optimization (PSO)}

Swarming strategies of bird flocking and fish schooling are used in the Particle Swarm Optimization introduced by Eberhart and Kennedy in 1995 [13, 14, 18, 28, and 29]. PSO is influenced by the simulation of social behavior rather than survival of the fittest as in the GA. Each individual benefits from its history and its interactions with other agents within the population. This sharing of knowledge helps facilitate faster convergence to an optimal solution. The choice of PSO parameters can have significant impact on optimization performance. Selecting PSO parameters that yield to proper performance has therefore been the subject of many researches [30, 31, and 32]. In this research, the controller tuning algorithm is based on Particle Swarm Optimization which is combined with the foraging behavior of $\mathrm{E}$ coli bacteria. The merge of the PSO and BF algorithms could result in better optimization strategy. PSO consists of swarming particles which are initialized with a population of random solutions. The particles move reiteratively through the solution space to search for the best solutions. Figure 6 shows a flowchart scheme of the PSO algorithm.

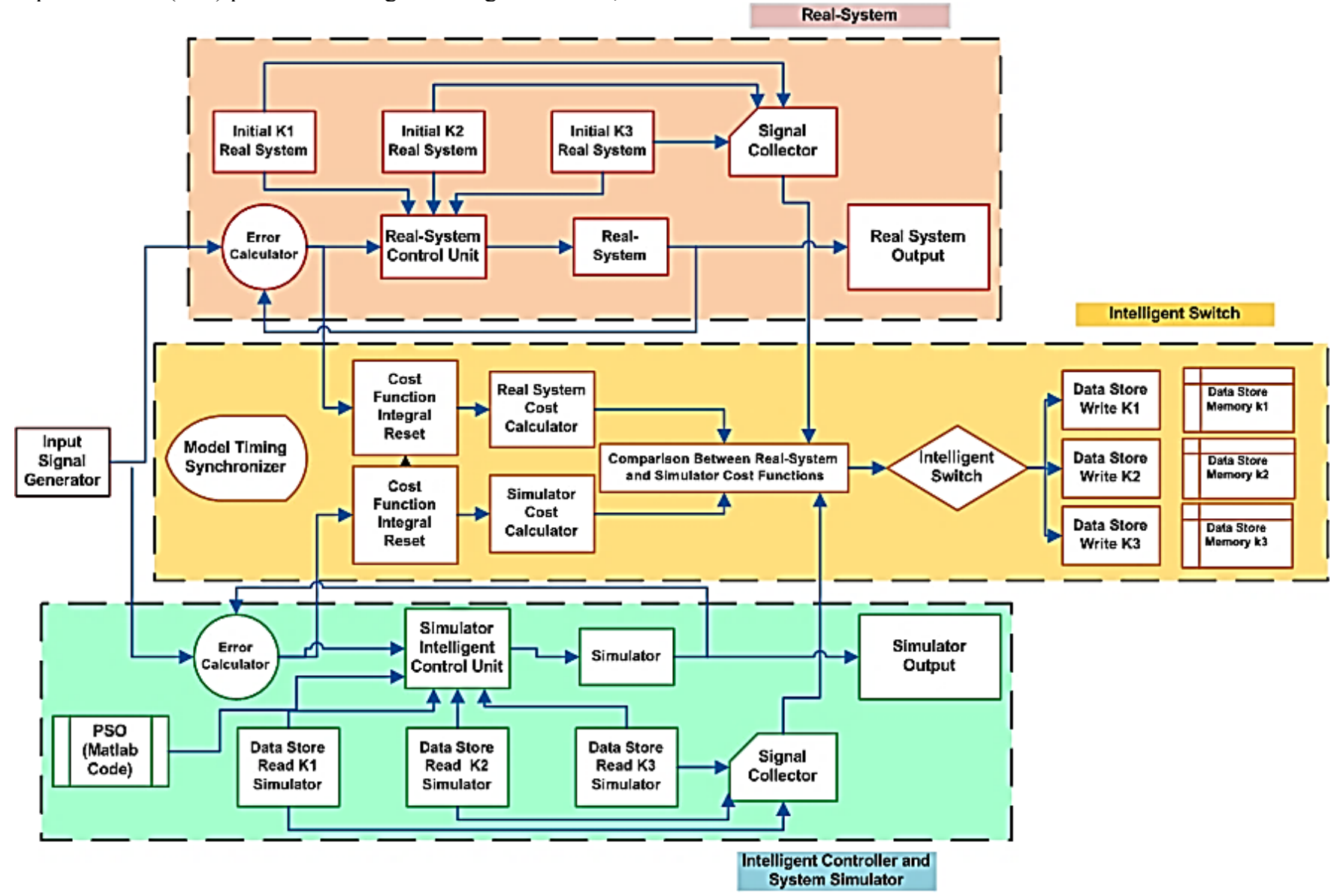

Figure 5: Structure of intelligent controller, simulator and intelligent switch in connection with the real system 


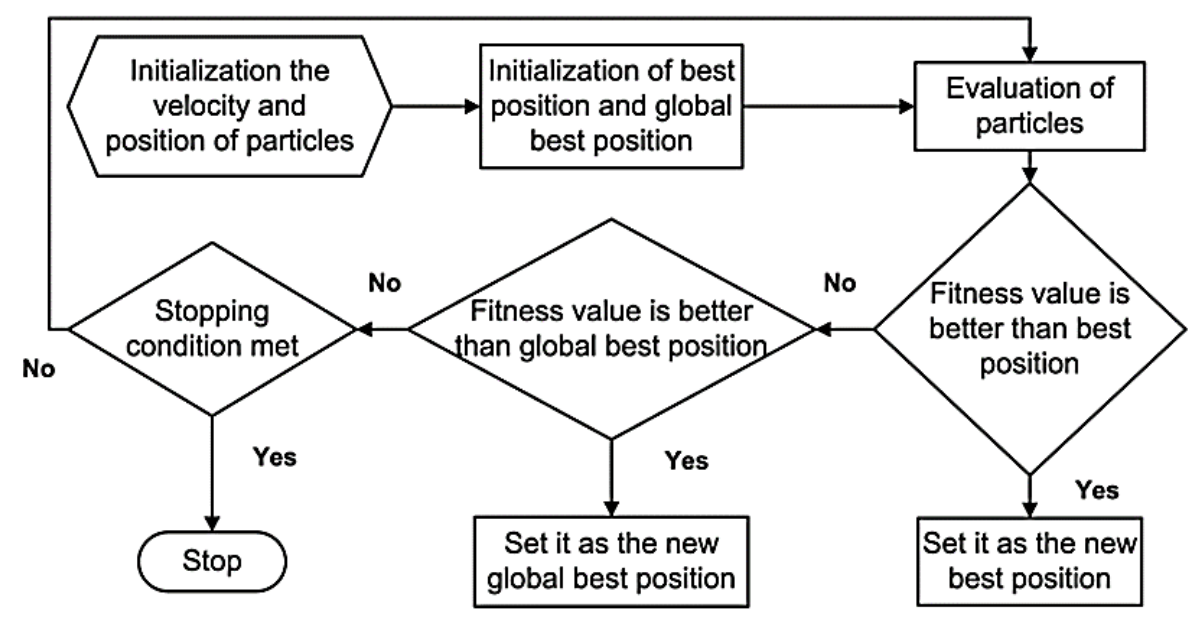

Figure 6: Scheme of PSO algorithm flowchart

The steps of the PSO algorithm are:

Step 1. Initialization of a number of particles; for a circuit component and random generation of a feasible value within the user-defined search range. The velocity is assigned by a random value in the range of maximum velocities.

Step 2. Calculation of the fitness value for each particle.

Step 3. Updating of the personal best position $\left(P_{b e s t}\right)$ for each particle according to its fitness value and updating of the global best position $\left(g_{\text {best }}\right)$ for the whole population.

Step 4. Updating of the position and velocity of every particle.

Step 5. Application of the mutation operator to enhance the population diversity. The operator is performed as follows: For every dimension of each particle, a random value is generated and is compared with the predetermined mutation probability.

Step 6. If the number of iterations exceeds the maximum value, the optimization process will be ended; otherwise it will return to Step 2.

Each particle has a position defined by a position-vector $X_{k}^{i}$, where $i$ is the index number of the particle, and its velocity is represented by velocity-vector $V_{k}^{i}$. Each particle recalls its own best position $P_{\text {Lbest }}$. Therefore the vector of the best position that comes from the swarm is saved in the vector of $P_{\text {Global. }}$.

During the iteration time $k$, the update of the velocity from the old velocity to the new velocity is defined by:

$$
V_{k+1}^{i}=V_{k}^{i}+R_{1}\left(P_{\text {Lbest }}^{i}-X_{k}^{i}\right)+R_{2}\left(P_{\text {Global }}^{i}-X_{k}^{i}\right)
$$

where $R_{1}$ and $R_{2}$ are random numbers.

A fresh position is calculated by utilizing the sum of former position vectors and the new velocity vector:

$X_{k+1}^{\stackrel{i}{+}}=X_{k}^{i}+V_{k}^{i}$

Every particle makes the decision to move to the future position by utilizing the data stored in the memory as the best position. The information about the most successful particle with the best position also affects the next particle position. This natural selection behavior of the particles improves foraging schemes and helps to prevent inappropriate foraging [33, 34].

The main goal of foraging process is to achieve the maximize energy per unit time which is spent for foraging after enormous generations. The $\mathrm{E}$ coli bacterium has a control system that enables it to search for best position and try to avoid noxious positions (local optimums).

In this research, the target is to find global minimal error integral when different input signals apply to the plant, which is equipped with controller by tuning the $\mathrm{K}_{1}, \mathrm{~K}_{2}$ and $\mathrm{K}_{3}$ control values.

The (BF-PSO) combines both algorithms BF and PSO. This combination aims to make use of PSO ability to exchange social information and BF ability in finding a new solution by elimination and dispersal which provides more sophisticated optimization algorithm.

PSO is based on recalling process, where past results are memorized and constantly improved until convergence is achieved. In comparison, neural-based methods such as Genetic Algorithms (GA), suffer from premature convergence since they rely on genetic operators that allow weak solutions to contribute to the composition of future candidate solutions [35]. The PSO algorithm permits all particles to have a quantum behavior instead of the classical Newtonian dynamics. Hence, instead of the Newtonian random walk, some sort of "quantum motion" is imposed in the search process.

When the PSO is tested against a set of benchmarking functions, it demonstrates superior performance as compared to the classical Newtonian methods under the condition of large population sizes. One of the most attractive features of the new algorithm is the reduced number of control parameters. Strictly speaking, there is only one parameter required to be tuned in the PSO.

\subsection{Bacterial Foraging Optimization (BFO)}

Bacterial Foraging Optimization (BFO) has been widely accepted as a global optimization algorithm of current interest for distributed optimization and control. In this method, the particle is making a decision to which position to move to the next position. The particle does this with attention to the previous data stored in the memory as the best past position. The data of the most successful particle with the best position also has effects on the next particle position. These selection behaviors of particles help to avoid poor foraging and improve foraging strategies. The main goal of the foraging process is to achieve the maximization 
energy per unit time which is spent for foraging after an enormous number of generations. The $\mathrm{E}$ coli behavior of the particle has a control system which makes it possible for it to seek out for the best position and try to stay away from local optimums (noxious substances).

Stage1-Swarming and Tumbling via flagella $\left(N_{s}\right)$ : The flagellum is a left-handed helix configured so that as the base of the flagellum (i.e. where it is connected to the cell) rotates counterclockwise from the free end of the flagellum looking toward the cell, it produces a force against the bacterium pushing the cell. This mode of motion is called swimming. Bacteria swim either for maximum number of steps $N_{s}$ or less, depending on the nutrition concentration and environment condition. However, if the flagellum rotates clockwise, each flagellum pulls on the cell, so that the net effect is that each flagellum operates relatively independently of the others and so the bacterium "tumble". Tumbling mode indicates a change in the future swim direction. Alternation occur between these two modes of operation in the entire lifetime.

Stage2-Chemotaxis $\left(N_{c}\right)$ : A chemotaxis step is a sequence of swim steps following a tumble. The maximum number of swim steps with a chemotactic step is predefined by $N_{s}$. The actual number of swim steps is determined by the environment [37].If the environment shows good nutrient concentration in the direction of the swim, the bacteria swim more steps. The end of the chemotactic step is determined by either reaching the maximum number of steps $N_{s}$ or by reaching a poor environment. When the swim steps stop, a tumble action takes place. To represent a tumble, a random unit length vector with direction $\operatorname{Delta}(n, i)$ is generated, where $n$ is the index for the chemotactic step, and $i$ is the index of the bacteria that has the maximum number of bacterium. This vector is used to define the direction of movement after a tumble. Let $N_{c}$ be the length of the lifetime of the bacterium as measured by the number of chemotaxis steps taken during its life. Let $c^{i}>0, i=1,2, \ldots, S$ denotes a basic chemotactic step size used to define the lengths of steps during runs where $S$ is the maximum number of bacteria. The step size is assumed to be constant.

The position of each bacterium is denoted by $P(n, i, j, k$, $\left.e_{l l}\right)$ where $n$ is the dimension of search space, $k$ is the index of reproduction step and $e_{l l}$ is the index of eliminationdispersal events.

The new bacterium position after tumbling is given by:

$$
P_{n, j+\stackrel{i}{1, k, e l l}}=P_{n, j, k, e l l}+\operatorname{Delta}(n, i) * c^{i}
$$

Stage3 - Reproduction $\left(N_{r e}\right)$ : Subsequent to $N_{c}$ steps, a reproduction process happens. $N_{r e}$ is the number of reproduction steps. For simplicity, it is assumed that $S$ is a non-negative number. Let:

$$
S_{r}=S / 2
$$

where $S_{r}$ is the number of population members who have had sufficient nutrients so that they will reproduce (split in two) with no mutations. In reproduction step, the bacteria population is sorted out to increase the collected population near the possible best global position. $S$ and $S_{r}$ are both positive integers [36].

For reproduction, the population is sorted in order of ascending accumulated cost (higher accumulated cost represents that it did not get as many nutrients during its lifetime of foraging and hence, is not as "healthy" and thus unlikely to reproduce). The $S_{r}$ least healthy bacteria die and the other $S_{r}$ healthiest bacteria each split into two bacteria, which are placed at the same location.

Stage 4: Elimination and dispersal $\left(N_{\text {ed }}\right)$ : Elimination event may occur for example when a local significant increase in heat kills a population of bacteria that are currently in a region with a high concentration of nutrients. A sudden flow of water can disperse bacteria from one place to another. The effect of elimination and dispersal events is possibly destroying chemotactic progress, but it also have the effect of assisting in Chemotaxis, since dispersal may place bacteria near good food sources.

The bacterial foraging algorithm has been tested for control applications like harmonic estimation for a signal distorted with additive noise, and for adaptive control. The combination of bacteria foraging and genetic algorithm is used to tune a controller of an automatic voltage regulator.

\subsection{Bacterial foraging oriented by Particle Swarm Optimization}

The BF-PSO combines both algorithms BF and PSO. This combination aims to make use of PSO ability to exchange social information and BF ability in finding a new solution by elimination and dispersal.

For initialization, the user selects $S, N_{s}, N_{c}, N_{r e}, N_{e d}, P_{e d}$, $C_{1}, C_{2}, R_{1}, R_{2}$ and $c(i), i=1,2 \ldots S$. Also position $P_{n}^{i}, \mathrm{i}=1$, 2 ... $S$ and velocity randomly initialized. The BF-PSO models bacterial Population Chemotaxis, swarming, reproduction, elimination and dispersal oriented by PSO is given below (Initially $j=k=e_{l l}=0$ ). Implicit subscribes will be dropped for simplicity. Figure 7 and the algorithm below it, illustrates the E-Coli flow diagram.

\section{Mathematical Model of the System}

\subsection{Valve and Hydraulic Actuator Model}

Electro hydraulic servo systems are commonly used in industry because of their high accuracy and large payload capacity. Modeling and control of such systems have been the focus of research for decades since models of these systems are often nonlinear and have parameters that are difficult to determine. The validity of models has usually been studied by approximate methods based on linearization methods, which do not unequivocally reveal the success of parameter estimation. Also system identification is a prerequisite for the analysis of such dynamic system.

The system under study (Figure 8) consisted of servo solenoid valve, cylinder, power unit, pressure sensors and displacement sensor. Several real-time simulation models for these systems have been proposed in previous research projects [19, 20, 21, and 22]. Table 1 illustrates the system specifications.

Table 1: The system specifications
\begin{tabular}{|c|l|c|c|}
\hline Notation & \multicolumn{1}{|c|}{ Note } & Value & Unit \\
\hline $\mathrm{A}_{1}$ & \multirow{2}{*}{ piston area } & $8.04 \times 10^{-4}$ & \multirow{2}{*}{$\mathrm{m}^{2}$} \\
\cline { 3 - 4 } & & $4.24 \times 10^{-4}$ & \\
\hline $\mathrm{A}_{2}$ & maximum stroke of the piston & 1 & $\mathrm{~m}$ \\
\hline$L$ & mass & 210 & $\mathrm{~kg}$ \\
\hline $\mathrm{m}$ & supply pressure & $14 \times 10^{6}$ & $\mathrm{~Pa}$ \\
\hline$p_{\mathrm{s}}$ & tank pressure & $0.3 \times 10^{6}$ & $\mathrm{~Pa}$ \\
\hline$p_{\mathrm{t}}$ & pipeline volumes at the two ports & $1.07 \times 10^{-4}$ & \multirow{2}{*}{$\mathrm{m}^{3}$} \\
\cline { 1 - 2 } & & $1.07 \times 10^{-4}$ & \\
\hline$v_{01}$ & & &
\end{tabular}




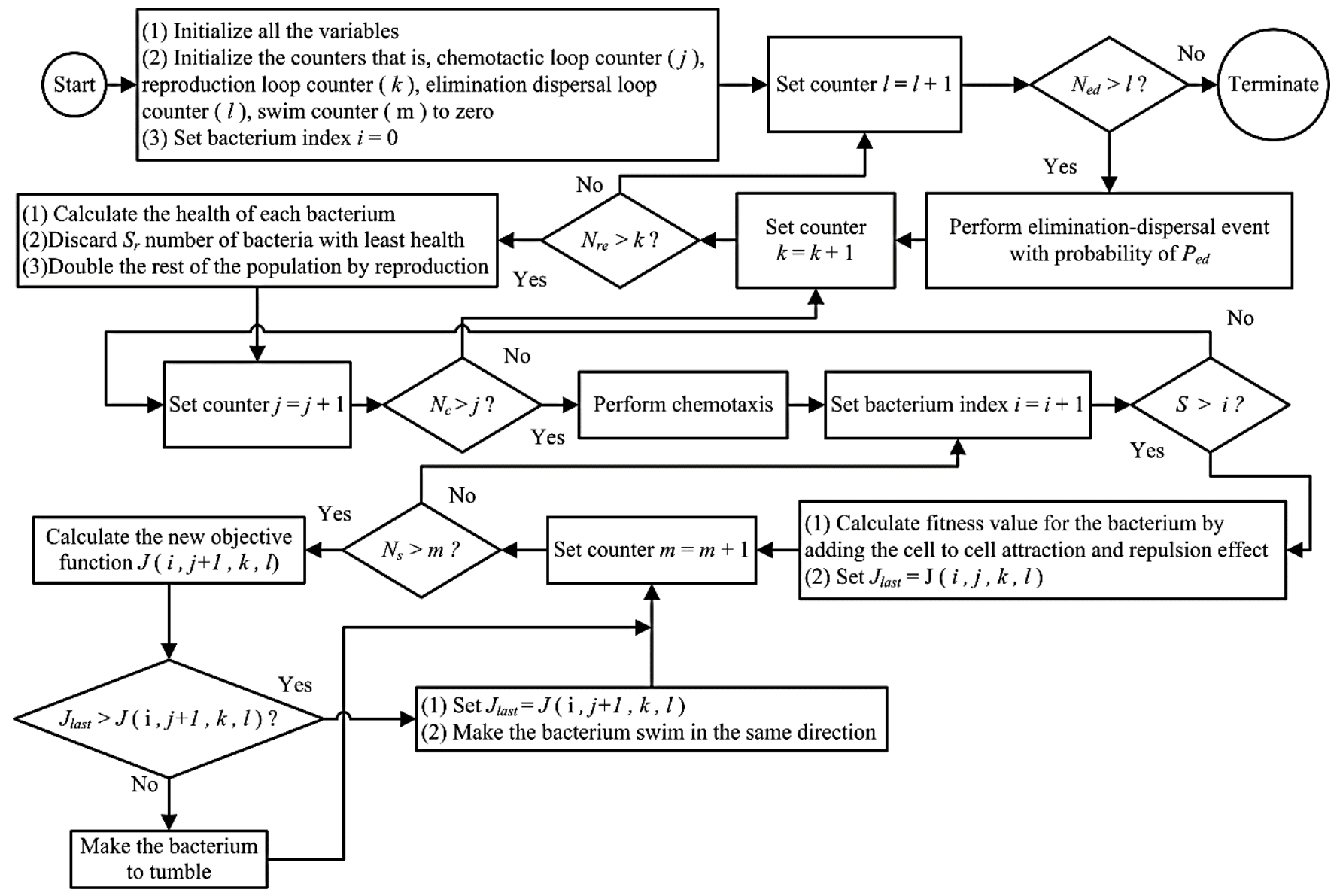

Figure 7: Schematic of an E-Coli flow diagram

The valve is a Bosch Rexroth servo solenoid valve with on-board electronics (4WRPET 6), having a nominal flow rate of $0.00067\left(\mathrm{~m}^{3} / \mathrm{s}\right)$. The data acquisition system is a dSPACE digital signal processor. The sampling frequency is $1000(\mathrm{~Hz})$. The control program is the $\mathrm{C} / \mathrm{C}++$ language program. The input voltage $u$ is fed to the valve using a DS $1103 \mathrm{I} / \mathrm{O}$ card; $u_{\mathrm{s}}$ is collected from the valve's Linear Variable Differential Transducer (LVDT) signal. The range of the LVDT signals $u_{s}(\mathrm{~V})$ is $\pm 10 \mathrm{~V}$ and $u_{s}$ is measurable. In this study, voltage $u_{s}$ is measured and directly used for providing information of the spool displacement. The system states, $p_{1}, p_{2}, p_{\mathrm{s}}, p_{\mathrm{t}}, x_{\mathrm{p}}$ are directly measured by pressure sensors and a displacement sensor, respectively. These sensors were calibrated by the respective manufacturers. When the input is applied to the valve, spool is shifted and openings are produced. The shift of the spool, namely position displacement $\mathrm{x}_{\mathrm{s}}(\mathrm{mm})$, is in both directions. The main spool of the valve is a mass held in position by a spring system. The main spool is the key component of the flow divider and is highly responsible for the outcome of the transfer function. A linearized model for an electro hydraulic servo system with a two-stage flow control servo valve and a double-ended actuator has revealed that the higher order model fits closer to the experimental data because of the reduced un-modelled dynamics. A first order model can be applied but the second order model responds the servo valve dynamics through a wider frequency range. When a second order transfer function is used to represent the valve model, the valve's dynamics could be as the following:

\section{Valve dynamics}

The standard second-degree valve's dynamic could be described as:

$$
\ddot{u}_{s}=k \cdot \omega_{n}^{2} \cdot u-2 \cdot \xi \cdot \omega_{n} \cdot \dot{u}_{s}-\omega_{n}^{2} \cdot u_{s}
$$

where $u$ is the input voltage to the valve, $u_{\mathrm{s}}$ is the collected signal from the valve's Linear Variable Differential Transducer (LVDT), $k$ is the gain, $\zeta$ is the damping ratio, and $\omega_{n}$ the natural angular frequency.

\section{Equation of motion}

The utilized actuator is a double acting hydraulic cylinder. Using the Newton's second law, the equation of motion for the servo hydraulic system becomes:

$m \cdot \ddot{x}_{p}=p_{1} \cdot A_{1}-p_{2} \cdot A_{2}-F_{f}$

Here, $m$ denotes the mass $(\mathrm{kg}), x_{\mathrm{p}}$ the displacement of piston $(\mathrm{m}), A_{1}$ and $A_{2}$ the piston areas $\left(\mathrm{m}^{2}\right), p_{1}$ and $p_{2}$ the pressures $(\mathrm{Pa})$ and $F_{f}$ the friction force $(\mathrm{N})$.

\section{Friction Force}

Friction force $\left(F_{f}\right)$ in the hydraulic cylinder is taken into account as an external disturbance. Friction is usually modeled as a discontinuous static mapping between the velocity and the friction force that depends on the velocity's sign. It is often restricted to the Coulomb and Viscous friction components. However, there are several frictional properties observed in the system, which cannot be explained by static models only. Examples of these complex properties are stick-slip motion, pre-sliding displacement and friction lag. The analytic model of friction dynamics, proposed by LuGre model, addresses all these characteristics of the friction. The motivation of using LuGre friction model is to have a friction model with higher accuracy that addresses the friction phenomena, which static models cannot fully explain. 


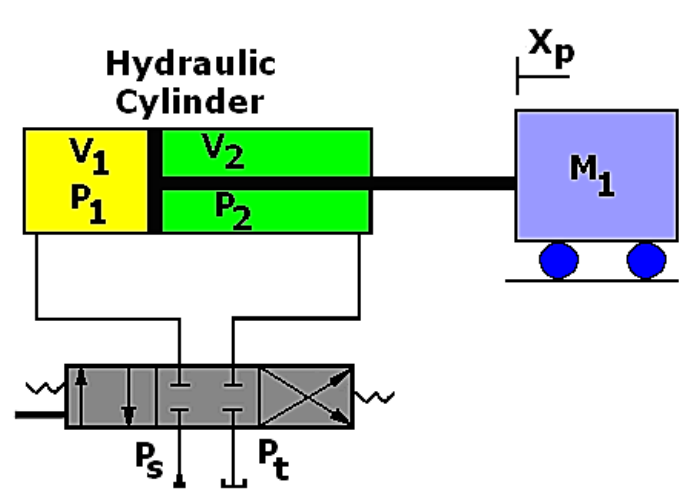

Figure 8: Schematic diagram of the servo hydraulic system (left) and the test bed used (right)

The model is defined by:

$$
\begin{aligned}
& F_{f}=\sigma_{0} \cdot z+\sigma_{1} \cdot \frac{d z}{d t}+k_{v} \cdot \dot{x}_{p} \\
& \frac{d z}{d t}=\dot{x}_{p}-\frac{\left|\dot{x}_{p}\right|}{g\left(\dot{x}_{p}\right)} Z
\end{aligned}
$$$$
g\left(\dot{x}_{p}\right)=\frac{1}{\sigma_{0}}\left[F_{c}+\left(F_{s}-F_{c}\right) \cdot e^{-\left[\frac{\dot{x}_{p}}{v_{s}}\right]^{2}}\right]
$$

where $z$ is an internal state, $g\left(\dot{x}_{p}\right)$ describes part of the "steady-state" characteristics of the model for constant velocity motions, $v_{s}$ is the Stribeck velocity, $F_{s}$ is the static friction, $F_{c}$ is coulomb friction, $k_{v}$ is the viscous friction, the stiffness coefficient is represented by $\sigma_{0}$ and damping coefficient by $\sigma_{1}[23,24]$.

\section{Valve flow}

The following equations describe the valve flows:

$$
\begin{array}{r}
Q_{1}=\left\{\begin{array}{l}
c_{s} \cdot u_{s} \cdot \operatorname{sign}\left(p_{s}-p_{1}\right) \cdot \sqrt{\left|p_{s}-p_{1}\right|, u_{s} \geq 0} \\
c_{s} \cdot u_{s} \cdot \operatorname{sign}\left(p_{1}-p_{t}\right) \cdot \sqrt{\left|p_{1}-p_{t}\right|}, u_{s}<0
\end{array}\right. \\
Q_{2}=\left\{\begin{array}{l}
c_{s} \cdot u_{s} \cdot \operatorname{sign}\left(p_{2}-p_{t}\right) \cdot \sqrt{\left|p_{2}-p_{t}\right|}, u_{s} \geq 0 \\
c_{s} \cdot u_{s} \cdot \operatorname{sign}\left(p_{s}-p_{2}\right) \cdot \sqrt{\left|p_{s}-p_{2}\right|}, u_{s}<0
\end{array}\right.
\end{array}
$$

with $c_{s}$ being the flow constant, $p_{s}$ the supply pressure and $p_{t}$ the tank pressure.

\section{Valve leakage}

The internal leakage flow is described as:

$$
Q_{L i}=L_{i} \cdot\left(p_{2}-p_{1}\right)
$$

In this equation, $L_{i}$ is the laminar leakage flow coefficient. The model of the external leakage flows in Eq. (13) was built as follows [5]:

$$
\begin{aligned}
& Q_{L 1}=L_{1} \cdot\left(p_{1}-p_{t}\right) \\
& Q_{L 2}=L_{2} \cdot\left(p_{2}-p_{t}\right)
\end{aligned}
$$

being $l_{1}$ and $l_{2}$ the laminar leakage flow coefficients.

\section{Pressure at the valve's ports}

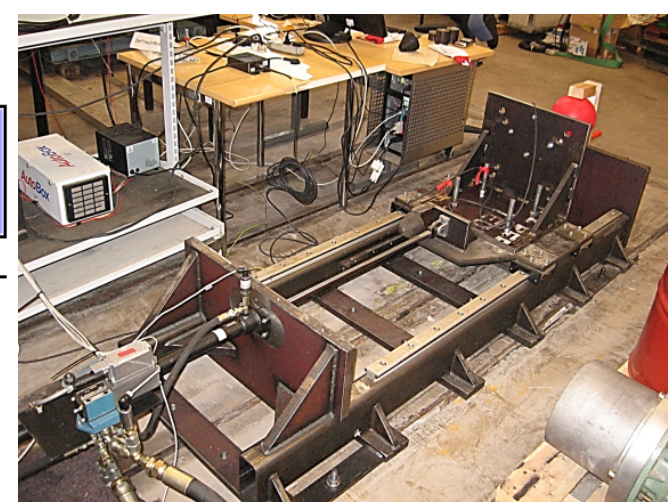

The pressures at the valve's ports are described as:

$$
\begin{aligned}
& \frac{d p_{1}}{d t}=\frac{\beta_{e 1}}{V_{1}}\left(Q_{1}-A_{1} \cdot \dot{x}_{p}+Q_{L i}-Q_{L 1}\right) \\
& \frac{d p_{2}}{d t}=\frac{\beta_{e 2}}{V_{2}}\left(-Q_{2}-A_{2} \cdot \dot{x}_{p}-Q_{L i}-Q_{L 2}\right)
\end{aligned}
$$

where $p_{1}$ and $p_{2}$ are the pressures at valve ports, $Q_{1}$ and $Q_{2}$ are the valve flows, $Q_{L i}$ is the internal leakage flow, $Q_{L l}$ and $Q_{L 2}$ are the leakage flows, $V_{l}$ and $V_{2}$ are the chamber volumes and $\beta_{e 1}$ and $\beta_{e 2}$ are the effective bulk modules of the cylinder. $\beta_{e l}$ and $\beta_{e 2}$ are represented by:

$\beta_{e i}=a_{1} \cdot E_{\max } \cdot \log \left[\left(a_{2} \cdot \frac{p_{i}}{p_{\max }}\right)+a_{3}\right]$

where $E_{\max }=1.8 \times 10^{9} \mathrm{~Pa}, p_{\max }=2.8 \times 10^{7} \mathrm{~Pa}$, and $a_{1}-a_{3}$ are coefficients of effective bulk modules.

\section{Chambers volume}

The volumes are calculated as:

$V_{1}=A_{1} \cdot x_{p}+v_{01}$

$V_{2}=A_{2} \cdot\left(L-x_{p}\right)+v_{02}$

where $v_{01}$ and $v_{02}$ are the pipeline volumes, and $L=1(\mathrm{~m})$ is the maximum stroke of the piston.

\subsection{Markov Chain Monte Carlo}

The mathematical model of the system involves a large number of parameters, which may be completely unknown or only known within certain ranges [25]. Markov chain Monte Carlo method is utilized to address these set of unknowns. In recent years, MCMC have emerged as a powerful tool for statistical analyses for nonlinear models. The MCMC technique has certain advantages in solving nonlinear problems, especially in obtaining probability distributions of parameters and model prediction, and allowing flexibility in the definition of the noise structure $[26,27]$. Statistical analysis studies the uncertainties in scientific inference by means of probabilistic reasoning. For the statistical treatment of uncertainties, it is assumed that all the unknown quantities can be described by statistical distribution, whether they are model parameters, unknown states of a system, model predictions or prior information of solutions. Typically, the state of the system is observed either directly or indirectly. A model is a mathematical description of the process that generates the states and the 
observations. The model can depend on a set of model parameters and it can be driven externally by control parameters, e.g., pressure. There is also a separate error model, which accounts for the unsystematic variation in the observations not covered by the systematic part of the model.

When the interest is in the model parameters, the inference is called parameter estimation. The related problem in applied fields is called the inverse problem. In inverse problems, the target of the estimation is an unknown function, describing the relationship between data and unknown parameters of the model in question. Statistically the unknown quantities are estimated with the help of the model, data, and a priori information about the unknown parameters. An electro hydraulic position servo system is under study and the MCMC approach is applied to model this nonlinear dynamic system [25]. After the initial analyses, it is noticed that the second order valve model is reliable, fits the dynamics of the used valve, and is chosen to describe the valve dynamics. The system model is finally constructed, including the nonlinearities of friction forces, valve dynamics, oil compressibility, load influence, the internal leakage, and the external leakage; the model parameters are identified. The model structure is developed until statistically acceptable results are achieved. The value of MCMC based model parameters are given in the following table. The MCMC model values are based on a research, which has been done on the same hydraulic system in Laboratory of Intelligent Machine of LUT by Jun-Hong Liu et al [25]. In such hydraulic system, some of the parameters would change during the working process. For instance, the oil viscosity will decrease while the temperature is increasing. The viscosity mainly has effects in laminar flow. In the hydraulic servo systems, the flow is mostly turbulent, thus viscosity plays a minor role in system behavior. The major effect this fact has is on the valve leakage, which may affect the system damping; as leakage increases the damping increases. On the other hand, the viscous friction acts as a counter effect; a smaller viscosity produces smaller viscous friction and damping. Nevertheless, in the simulator model the viscosity is always constant. However, the model is accurate enough and follows the real system.

\subsection{Real-time simulator validity}

To test the validity of the simulator (based on a second degree servo system), three independent physical experiments were carried out, and they were different from each other by the input value, the mass load, and/or the supply pressure (Figure 9). The reason of using three different valve inputs in three different loadings was to check the stability of the model in different situations. The observations, namely $x_{p}, p_{1}, p_{2}, u_{s}$ and control parameters, explicitly $u, p_{s}, p_{t}$ were directly collected along with time in each experiment. The response of the identified model matches the observations in each case [25]. Since the simulator has been verified by different inputs, it has same response with real system within bandwidth of the system.

\section{Intelligent Switch algorithm}

The main responsibility of the switch is to feed the real system with the best available control values. As mentioned earlier, the real system is equipped with the linear controller and the simulator is equipped with the intelligent controller. By applying the same reference input into both systems, in every iteration, the switch calculates the cost value of both systems.

\begin{tabular}{|c|c|c|c|}
\hline Notation & Note & Value* $^{*}$ & Unit \\
\hline$a_{1}$ & \multirow{3}{*}{$\begin{array}{l}\text { coefficients of effective } \\
\text { bulk modulus }\end{array}$} & 0.3102 & \multirow{3}{*}{$\begin{array}{l}\text { (no unit) } \\
\text { constant }\end{array}$} \\
\hline$a_{2}$ & & 49.18 & \\
\hline$a_{3}$ & & 1.843 & \\
\hline$c_{\mathrm{s}}$ & flow constant & $3.021 \times 10^{-8}$ & $\mathrm{~m}^{3} \mathrm{~s}^{-1} \mathrm{v}^{-1} \mathrm{~Pa}^{-1 / 2}$ \\
\hline$F_{\mathrm{C}}$ & Coulomb friction level & 74.81 & $\mathrm{~N}$ \\
\hline$F_{\mathrm{S}}$ & $\begin{array}{l}\text { static friction force } \\
\text { level }\end{array}$ & 2921 & $\mathrm{~N}$ \\
\hline$k$ & gain & 0.9907 & No unit \\
\hline$k_{\mathrm{v}}$ & $\begin{array}{l}\text { viscous friction } \\
\text { coefficient }\end{array}$ & 87.74 & \\
\hline$l_{1}$ & \multirow{4}{*}{$\begin{array}{l}\text { Leakage flow } \\
\text { coefficients }\end{array}$} & $1.038 \times 10^{-13}$ & \multirow{4}{*}{$\begin{array}{l}\mathrm{Ns} / \mathrm{m} \\
\mathrm{m}^{3} \mathrm{~s}^{-1} \mathrm{~Pa}^{-1}\end{array}$} \\
\hline$l_{2}$ & & $8.485 \times 10^{-13}$ & \\
\hline$l_{3}$ & & $5.422 \times 10^{-13}$ & \\
\hline$l_{4}$ & & $1.623 \times 10^{-13}$ & \\
\hline$L_{\mathrm{i}}$ & $\begin{array}{l}\text { laminar leakage flow } \\
\text { coefficient }\end{array}$ & $1.19 \times 10^{-12}$ & \\
\hline$u_{\mathrm{s} 1}$ & \multirow{4}{*}{$\begin{array}{l}\text { input voltages for the } \\
\text { individual maximum } \\
\text { leakage openings }\end{array}$} & $1.964 \times 10^{-5}$ & \multirow{4}{*}{$\begin{array}{c}\mathrm{m}^{3} \mathrm{~s}^{-1} \mathrm{~Pa}^{-1} \\
\mathrm{~V} \text { (Voltage) }\end{array}$} \\
\hline$u_{\mathrm{s} 2}$ & & -0.6993 & \\
\hline$u_{\mathrm{s} 3}$ & & -0.1123 & \\
\hline$u_{\mathrm{s} 4}$ & & 9.967 & \\
\hline$v_{\mathrm{s}}$ & Stribeck velocity & 0.1624 & \\
\hline$\zeta$ & damping ratio & 0.5588 & $\mathrm{~m} / \mathrm{s}$ \\
\hline$\sigma_{0}$ & $\begin{array}{l}\text { flexibility coefficient of } \\
\text { friction force }\end{array}$ & 1521 & $\begin{array}{l}\text { no physical } \\
\text { unit }\end{array}$ \\
\hline$\sigma_{1}$ & $\begin{array}{l}\text { damping coefficient of } \\
\text { friction force }\end{array}$ & 848.3 & $\mathrm{~N} / \mathrm{m}$ \\
\hline$\omega_{\mathrm{n}}$ & $\begin{array}{l}\text { natural angular } \\
\text { frequency }\end{array}$ & 481.3 & $\mathrm{Ns} / \mathrm{m}$ \\
\hline
\end{tabular}

If the intelligent controller prepares better results in comparison with the real system controller, then it will control both systems for one iteration and update the real system controller with the new control values. In the next iteration the output of both systems will be checked via the switch again and if the intelligent controller does not provide better results than the real system will switch back to the linear controller. If the error in any time step become large then the integral effect may become significant. In order to avoid this problem the algorithm benefits from forgetting factor as well. The designed switch uses the Integral of the Squared Error (ISE) cost function to find out which control values, from the linear controller or the intelligent controller, produces a lowest ISE value. There are different cost functions to find the best control values but the squared error integral criteria is the most common for such optimization.

One of the most important issues when switching from linear controller to the intelligent control values is to avoid of applying any shock in the real system because of the new control values. In order to solve this problem the new control values are applied to the real system by using a smoothing function. The smoothing function is a function between the old and new control values. The function provides a bridge between old and new control values. By using this method, the new control values are applied to the system smoothly to avoid any shock into the real system.

\subsection{Optimization Convergence}

It is highly desirable that iterative algorithms for solving optimization problems converge fast to solutions that are global minimizers of the objective function. The rate of convergence is one of the important features that distinguishes one optimization method from another. PSO 
has several attractive features that make it an ideal candidate for controller tuning namely, fast convergence, a simple and
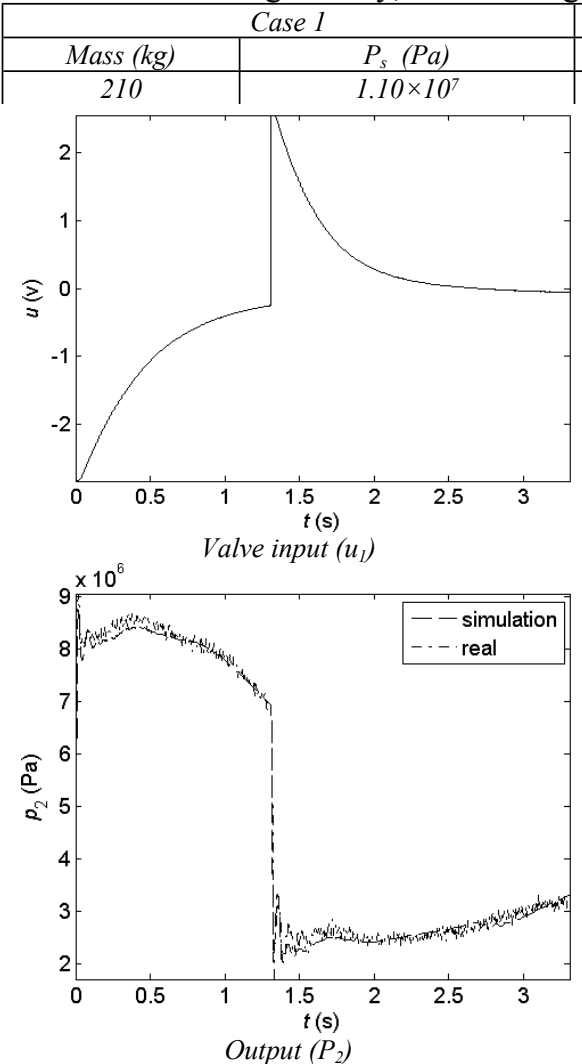

efficient operating algorithm, and repeatability.

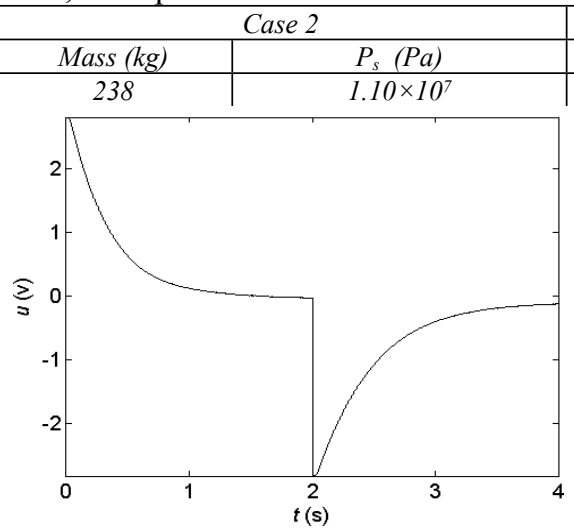

Valve input $\left(u_{2}\right)$

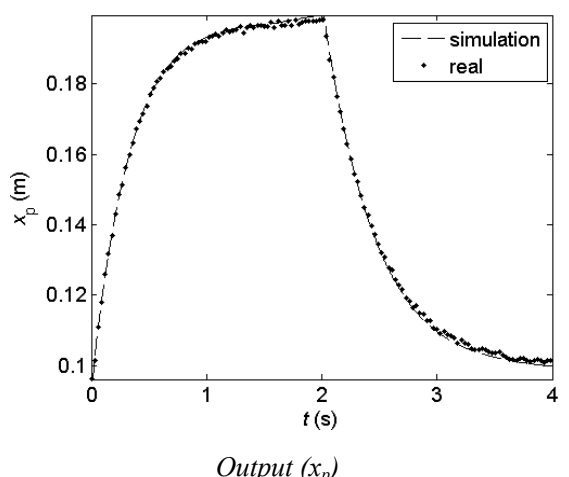

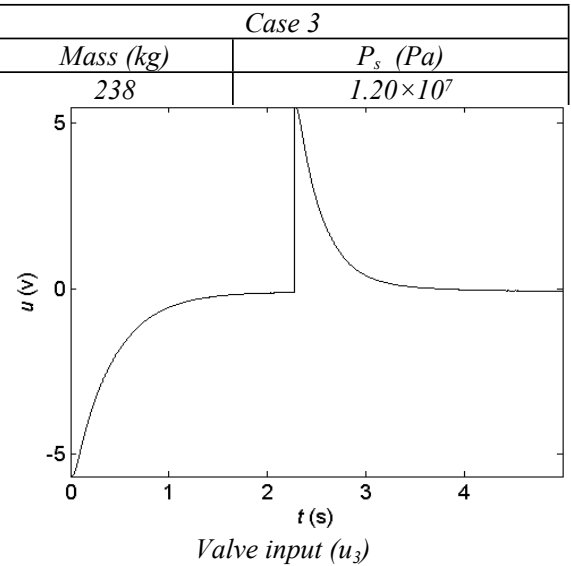

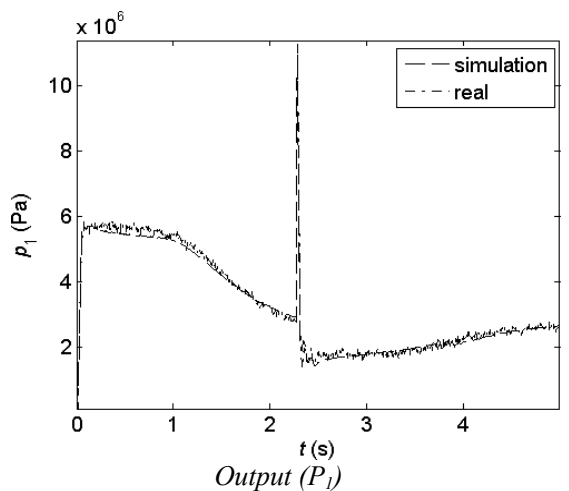

Figure 9: Verification of identified model's stability using experiments with different control signals, loads and pressures [25]

In this study, the convergence rate of the optimization was tested in the case of a pulse input to find out how many optimization cycles are needed to minimize the cost function. Figure 10 illustrates the random search behavior of the PSO algorithm in the search space and finally its convergence. Based on the random search behavior of the optimization and its progress to a converged point, the simulator output to a pulse input is illustrated in Figure 11. As shown in Figure 11, low quality generations of control parameters should be filtered out from the solution space.

A filtration algorithm is designed to reject bad generations that are produced because of the random search behavior of utilized algorithm. This algorithm collects and saves better generation of solutions from the search space. Figure 12 shows the cost function values after filtering bad generations of answers.

\section{Stability}

\subsection{Stability control}

In this study, it is hard to prove the global robustness of the system because of direct random search behavior of the controller. In utilized control strategy, the control parameters are optimized on each iteration. It means that in order to check the system robustness the Lyapunov Stability Criteria should be calculated to check the stability in every iteration. The Lyapunov criteria is designed based on fixed control values. But the control parameters in this study are almost changing in each iteration. The solution is to check the system stability under real disturbances. The proposed control method is designed to act well as long as uncertain parametric quantities or disruptions are within some set. The method aims to achieve stability in the existence of delimited modeling errors.

\subsection{Pressure disturbance}

In first set of stability tests the supply pressure of the hydraulic circuit is decreased to the $50 \%$ of the value of hydraulic pressure input in the control block. Figure 13 illustrates the system output in presence of pressure drop. The red curve is the input, the green curve is the simulator output and the blue curve is the real system output. With attention to the Figure 13, the real system has some offset from the reference signal.

However, in next iterations the control system affects positively and pushes the real system to follow the reference signal with less error. As it is shown in this figure, the intelligent controller is converged almost immediately. In comparison, the controller needs more time to converge in existence of pressure disturbance.

The response of the same system to a sine wave input after convergence is shown in Figure 14. After few seconds, both systems have the minimum error from the input.

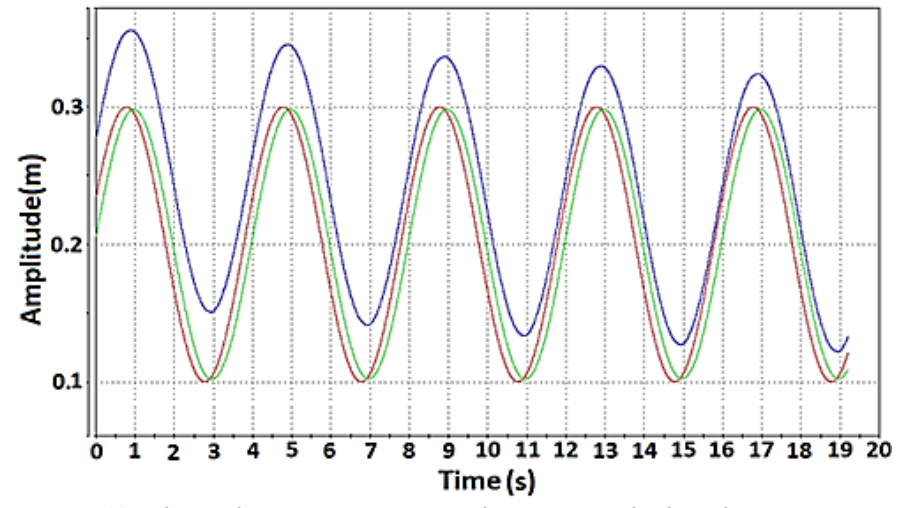

Figure 13. The real system response to the sine signal when the pressure disturbances applied to the system 

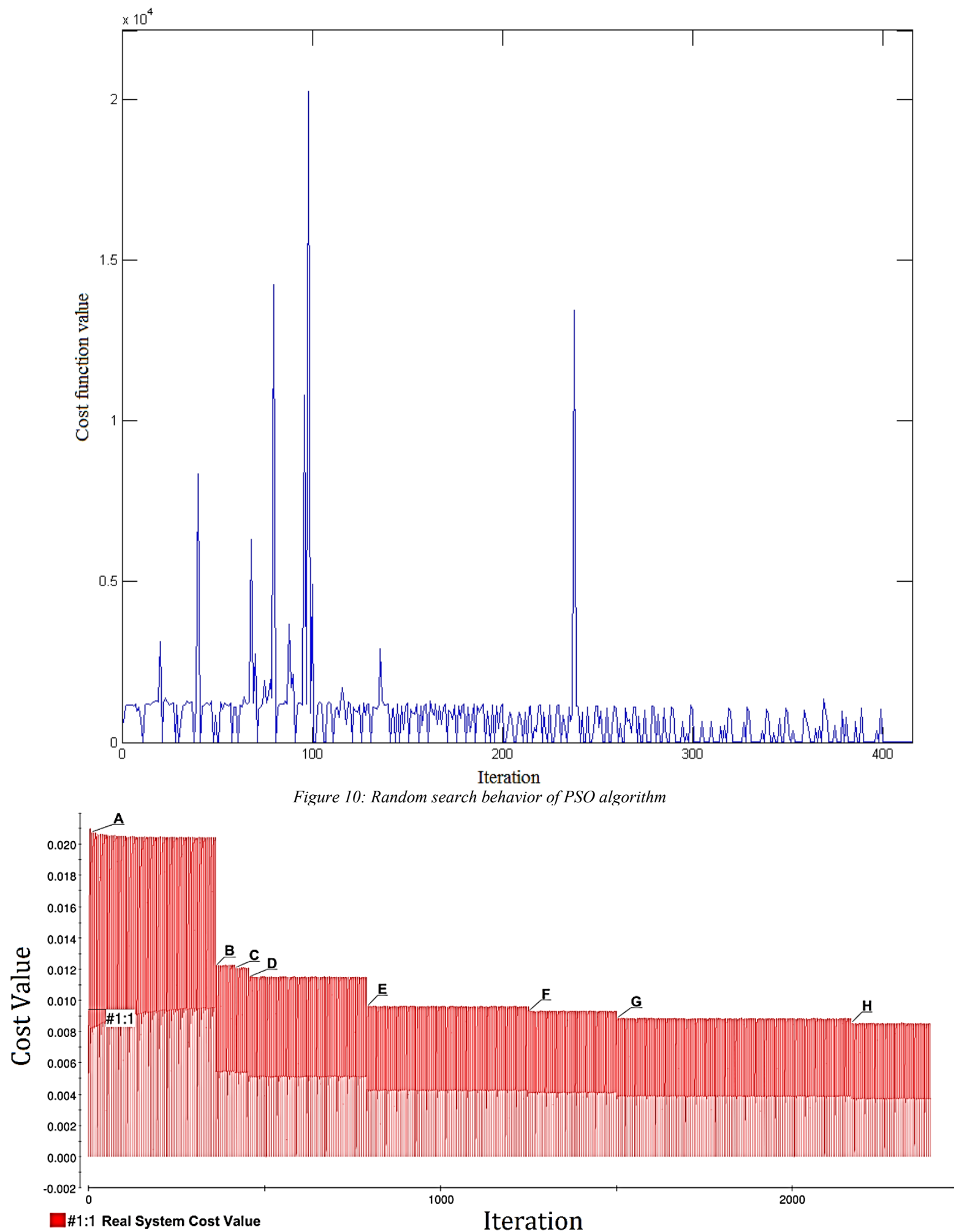

Figure 12: Optimization Convergence

Table 3: Control parameters based on optimization convergence

\begin{tabular}{|c|c|c|c|c|}
\hline & $\mathrm{K}_{1}$ & $\mathrm{~K}_{2}$ & $\mathrm{~K}_{3}$ & $\begin{array}{c}\text { Max Real } \\
\text { System cost } \\
\text { value end } \\
\text { of single } \\
\text { iteration }\end{array}$ \\
\hline A & 33.000 & 0.300 & 0.030 & 0.02079 \\
\hline B & 55.664 & 0.352 & 0.046 & 0.01235 \\
\hline C & 64.701 & 0.490 & 0.008 & 0.01201 \\
\hline D & 83.229 & 0.376 & 0.070 & 0.01189 \\
\hline E & 112.819 & 0.409 & 0.079 & 0.00981 \\
\hline F & 120.018 & 0.639 & 0.008 & 0.00938 \\
\hline G & 126.429 & 0.451 & 0.118 & 0.00882 \\
\hline
\end{tabular}



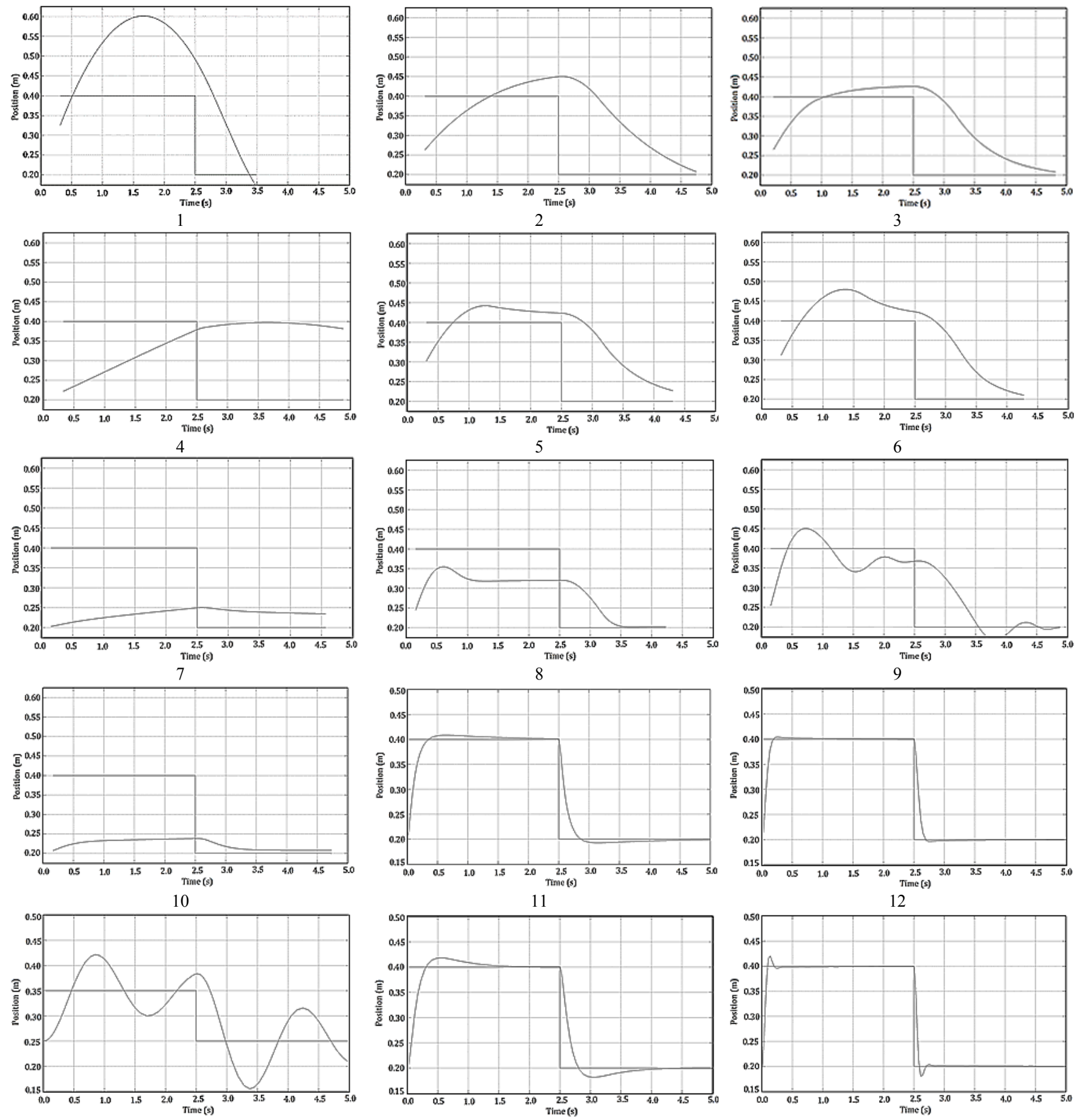

13
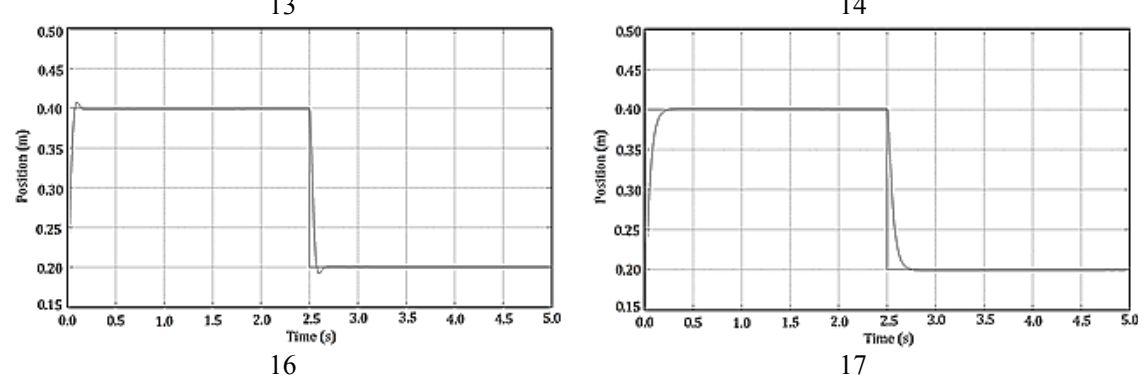

Figure 11. Random search behavior of PSO algorithm in the space of answers

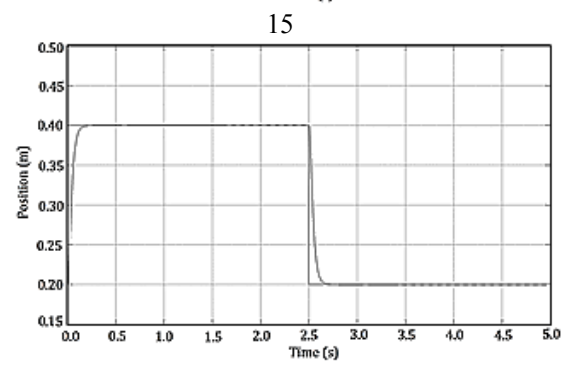




\subsection{Mass disturbance}

It is difficult to measure the load disturbance under normal circumstances. Figure 15 illustrates the real system output to the ramp input when mass disturbance applied into the system. As it is shown in Figure 15, the adaption is slow and takes almost 10 seconds.

The reason of this delay is that the mass was changing randomly until 20 seconds and then fixed (a lab technician was standing on the slider). The supply pressure is $50 \%$ of the max supply pressure. In first cycles the system is far from the ramp input signal but after few iterations the controller pushes the real system to better output with less error.

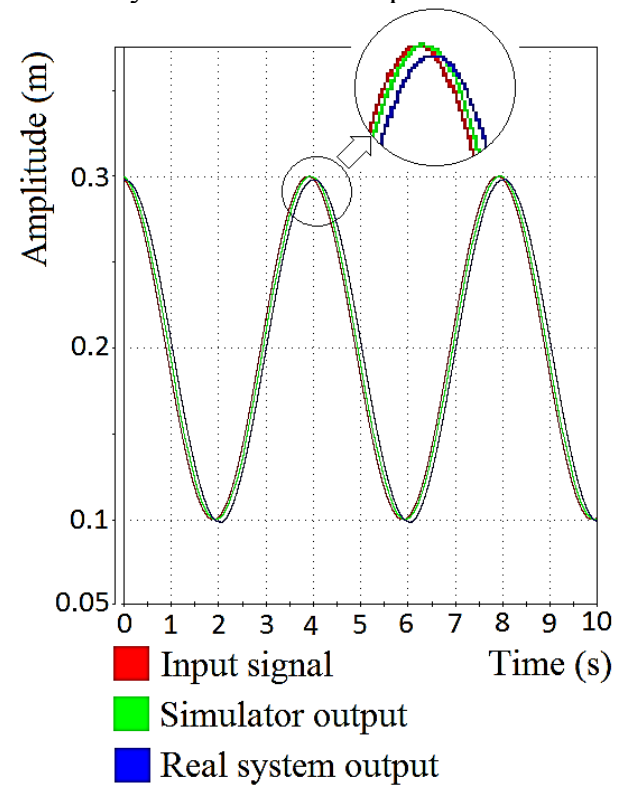

Figure 14. Shows the system input and outputs after few seconds when the error is in its minimum value

\subsection{Force Disturbance (Haptic Test)}

In this set of tests, the joystick controlled the hydraulic slider, to which a force sensor was mantled on the end of the actuator. The transducer squeezed a test ball. Figure 16 and 17 illustrates the structure of teleoperation controller [17].

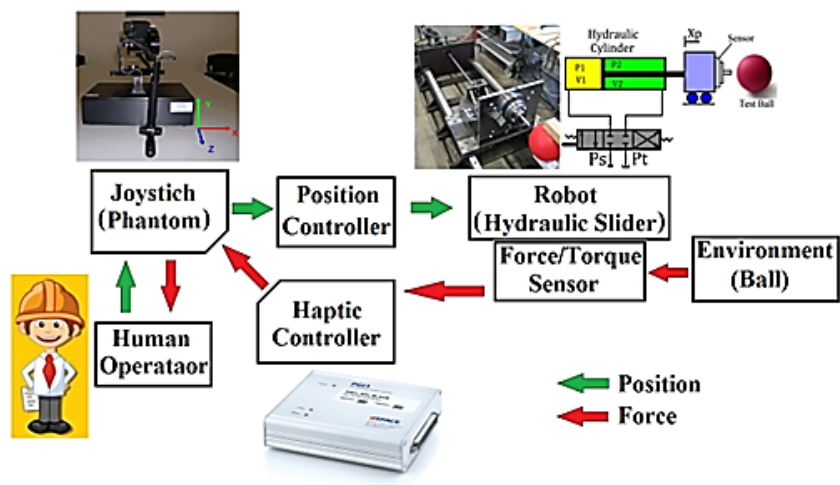

Fig. 16. Structure of teleoperation controller

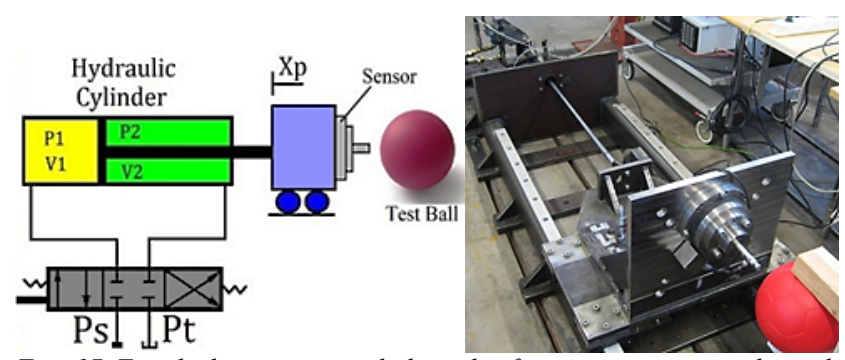

Fig. 17 Test bed structure including the force sensor mounted on the
The haptic device used in this study, is PHANTOM premium 1.5, 6 DOF, built up by SensAble Technologies, Inc. The PHANTOM system has been widely recognized as a reliable 3 degree-of-freedom (3DOF) force-feedback device available. The objective is to achieve a high level of positioning accuracy with a haptic device. PHANTOM devices have good inherent repeatability under no-load conditions. However, they can only be accurate if they have been initialized properly. Workspace coordinates for all PHANTOM haptic devices are specified in the Cartesian coordinate system. By default, the positive $\mathrm{X}$-axis points to the right of the PHANTOM, parallel to the front plate; the positive $\mathrm{Y}$-axis points up; and the positive $\mathrm{Z}$-axis points "out". Figure 18 illustrates the Cartesian Device Space for PHANTOM 1.5 6DOF [38].

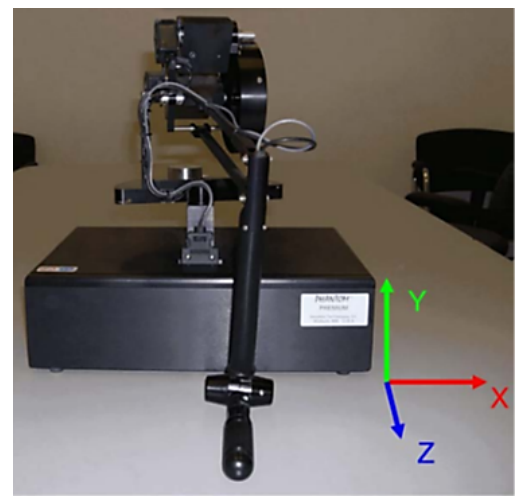

Figure 18: Cartesian Device Space for PHANTOM 1.5 6 DOF

PHANTOM is equipped with incremental rotary encoders that evaluate the joint angles of the device. These angle measurements are used to calculate the end effector position in Cartesian space. Table 4 presents the specifications of the device [34].

Table 4: PHANTOM Premium 1.5 F specifications [35]

\begin{tabular}{ll}
\hline \hline Feature & Specification \\
\hline Workspace & $381 \mathrm{~W} \times 267 \mathrm{H} \times 191 \mathrm{D}(\mathrm{mm})$ \\
Back drive friction & $0.75 \mathrm{oz}(0.2 \mathrm{~N})$ \\
Continuous exert able force & $1.4 \mathrm{lbf}(6.2 \mathrm{~N})$ \\
Inertia without encoder gimbal & $<0.33 \mathrm{lbm}(<150 \mathrm{~g})$ \\
Position Sensing & $\mathrm{x}, \mathrm{y}, \mathrm{z}($ roll, pitch, Yaw $)$ \\
Footprint & $330 \mathrm{~W} \times 254 \mathrm{D}(\mathrm{mm})$ \\
Nominal Position Resolution & $3784 \mathrm{dpi}(0.007 \mathrm{~mm})$ \\
Maximum exert able force & $8.4 \mathrm{lbf}(37.5 \mathrm{~N})$ \\
Stiffness & $20 \mathrm{lbf} \mathrm{in}{ }^{-1}\left(3.5 \mathrm{~N} \mathrm{~mm}^{-1}\right)$ \\
\hline \hline
\end{tabular}

OMEGA 160 [36] is the force sensor utilized in this study. The sensor measures the full six components of force and torque using a monolithic instrumented transducer. The sensor measures all six components of forces and torques from all three Cartesian coordinates $(x, y$ and $z$ ).

The specification and sensing range of the transducer are given in Table 5. The sensor is well calibrated by its manufacturer, ATI Industrial Automation Co, before starting the tests. In order to avoid typical gain errors caused by temperature fluctuations, all the reference readings are taken at the same temperature before and after applying the load of interest [37]. The tele-operated electrohydraulic servo system is controlled via a force feedback joystick, which provides haptic capabilities for the operator. 


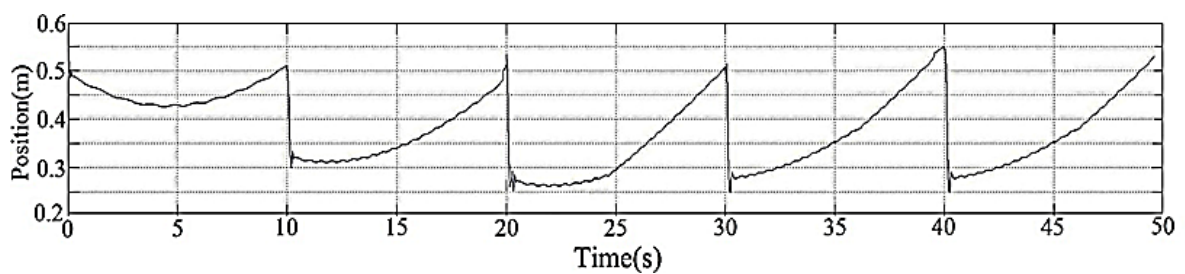

Figure 15: The real system ramp response when the mass disturbance is applied to the system

Table 5: OMEGA 160 IP60 specifications, range and resolution [36]

\begin{tabular}{l|ll}
\hline \hline \multirow{2}{*}{ Sensing range } & ${\text { Max } \mathrm{F}_{\mathrm{x}}, \mathrm{F}_{\mathrm{y}}} \pm 2500 \mathrm{~N}$ \\
& $\operatorname{Max~T}_{\mathrm{x}}, \mathrm{T}_{\mathrm{y}}$ & $\pm 400 \mathrm{~N}-\mathrm{m}$ \\
\hline \multirow{3}{*}{ Physical specifications } & Weight & $7.67 \mathrm{~kg}$ \\
& Diameter & $190 \mathrm{~mm}$ \\
& Height & $58 \mathrm{~mm}$ \\
\hline \hline
\end{tabular}

Haptic devices are capable of measuring bulk or reactive forces that are applied by the user into the interface. In order to control force with a position-based system, a precise model of the mechanism and knowledge of the exact location of the environment are required. With the use of force feedback from the interaction, that takes place; very litter model information is required in order to close the loop around the contact force [7]. In this project the target is to control the tele-manipulation system so that it looks active to the work environment and the human operator as if they are both getting together with a common practical rigid mechanical instrument. The control strategy allows the human to be kinesthetically and dynamically, plugged into its work environment. The teleoperation controller, which controls both the valve and the motorized joystick, is designed for sufficiently slow manipulation, achieves asymptotic coordination of the joystick and the hydraulic actuator. The simple diagram of the closed loop tele-operator is shown in Figure 19. The controller is a new class of modelbased control algorithm. The new control algorithm provides asymptotically exact tracking of both the position and the contact force.

In this study the force feedback treated as a disturbance force. The amount of this disturbance is measured accurately via the force sensor and updated in the system model. Then the system stability is checked when this force existing due to the contact between the force sensor and the environment.

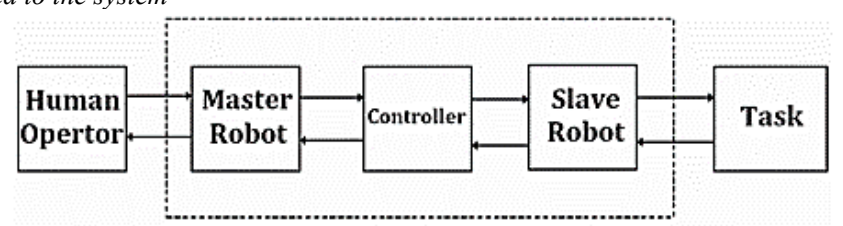

Figure 19: Interaction of teleoperation

In this experiment, a simple contact which in first part the slider goes forward to touch the ball and in second part the slider goes back to its stationary point. Figure 20 illustrates the sensor mounted on the slider and while squeezing the test ball.

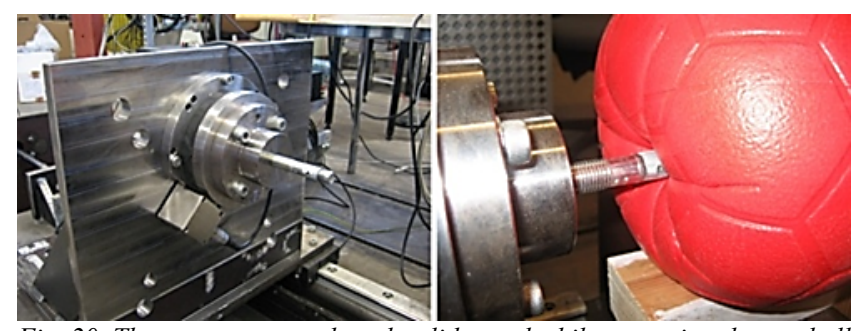

Fig. 20 The sensor mounted on the slider and while squeezing the test ball

Figure 21-a illustrates the force-position curve in this test. In the upper curve the slider starts to move into the ball. After reaching to the maximum safe point, the slider pushed back to the zero point. The second part of the movement which the slider pushed back is illustrated in the lower curve of Figure 21-a. Figure 21-b shows the position of the slider and PHANTOM in this experiment. These experiments prove that the introduced control strategy provides stability for the real system in existence of disturbances.

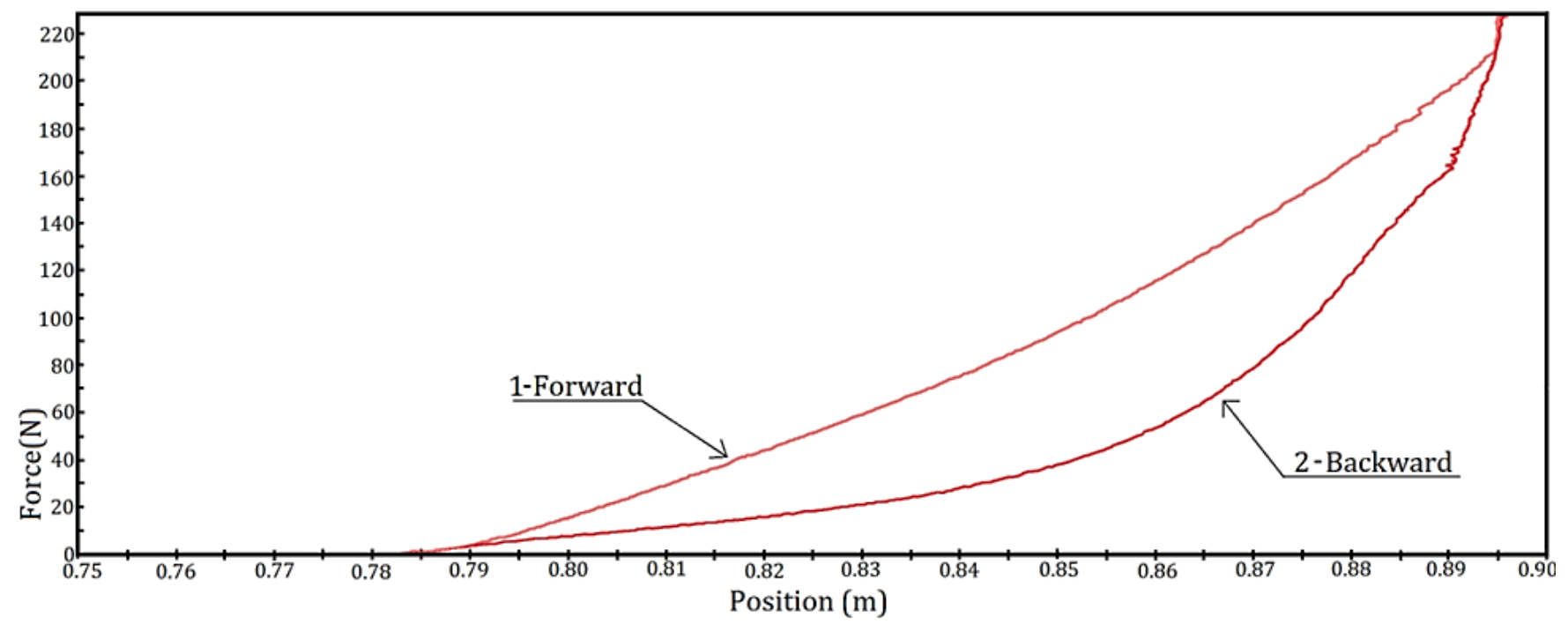

(a) 


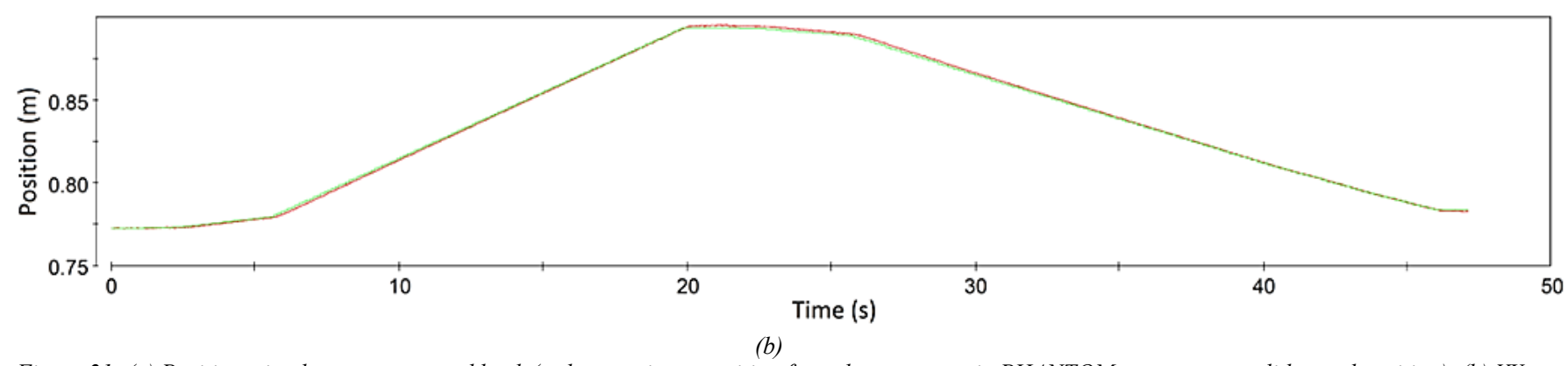

Figure 21: (a) Position, simple contact, go and back (red curve: input position from the operator via PHANTOM, green curve: slider real position), (b) XY plot, Position-Force, simple contact, forward and backward movement

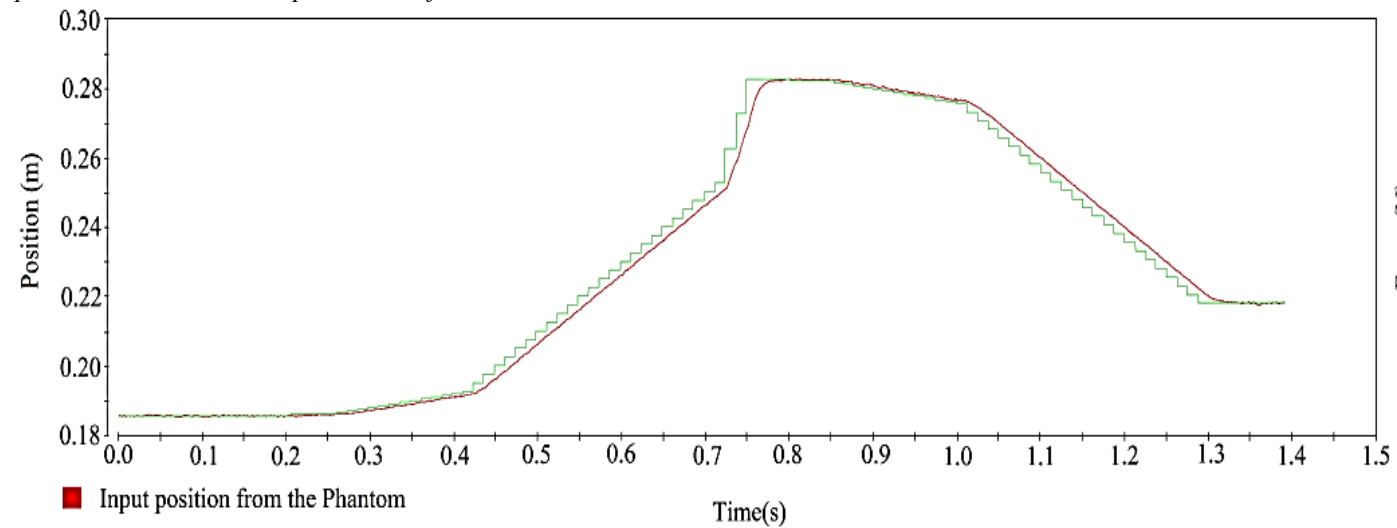

- Slider position

(a)

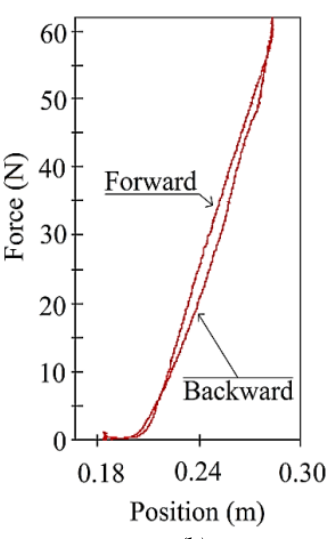

(b)

Figure 22: (a) Position of the slider in response to the input position from the Phantom $\quad$ (b) Position-Force curve in a simple contact

As it is illustrated, in existence of disturbance force, the slider follows the input position. The amount of force which the operator feels in his/her hand via the Phantom is a $35: 1$ scaled force sensed by the force sensor. It means that the form of the haptic force is the same as it is shown in Figure 21-a. It should be mentioned that the maximum amount of force which a Phantom premium can stand is $7 \mathrm{~N}$. The reasons for the difference between the forces shown on upper and lower curves in Figure 18-a are: 1) Sensor hysteresis. 2) The different behavior of the ball when it is squeezed and when it is released. 3) The operator hands behavior.

\subsubsection{HIGH FREQUENCY TEST}

Figure 21 shows a simple and very slow movement; approximately $5 \mathrm{~cm}$ in 10 seconds. In order to test the controller performance on much faster movement the test is repeated in a speed close to human speeds (almost $10 \mathrm{~cm}$ in one second). The result is illustrated in Figure 22.

In Figure 22-a the red curve is the position input from the Phantom and the green curve is the position of the slider.

As it is shown in Figure 22, the controller performs well under faster movements. There are some errors in this kind of movement but as the amount of error is small and the movement is fast then this error is ignorable.

The curve (b) is the force-position curve when the ball is squeezed and the right-hand curve is the force-position curve when the ball released. The hysteresis effect is obvious in this figure. The operator fills the same force function in his hand with a $35: 1$ scaling decrease.

\section{Conclusion}

This study proposes a novel control method for a teleoperated electrohydraulic servo system. This methods implements a position control and haptic sense between the human and manipulator interaction, and between the manipulator and task environment interaction. The

proposed method has the characteristics of a universal technique, independent of the actual control algorithm, and it can be applied with other suitable control methods as a real-time control strategy.

In this study, the control algorithm is based on PSO, combined with the foraging behavior of $E$. coli bacteria. As the proposed strategy is globally valid, it is possible to replace the PSO algorithm with other optimization strategies. This study has shown that using the PSO methodology is characterized by an improved control behavior for set point tracking and disturbance rejection. PSO has several attractive features that make it an ideal candidate for controller tuning, namely: fast convergence, a simple and efficient operating algorithm, and repeatability. The results show that the performance of the proposed control strategy is very stable and has better response than a conventional controllers.

The PSO is influenced by the simulation of social behavior in which each individual benefits from its history and its interactions with other agents within the population. This sharing of knowledge helps facilitates faster convergence to an optimal solution. The use of simple mathematical operators facilitates a faster computational time and makes the algorithm suitable for determining tuning parameters under high-speed dynamical conditions for processes that lend themselves to tuning of this nature, such as flow and pressure control. From the tests that were conducted, it was shown that the PSO parameters provide the best control performance; this is evident from the low ISE that was observed during the tests. Tuning parameters obtained with the PSO are consistent over a number of tuning sessions. This does not apply to the GA based tuning method. From the results presented in the study it was shown that, the PSO tuning yielded improved responses and can be applied to different process models encountered in the process control industry. 
Previous research projects have shown that directed random search methods such as GE and DE algorithms have serious drawbacks, such as unstable generations and slow convergence. In addition, their practical application is restricted because of the risk of damage caused by their instability. Most neural controllers use feed-forward type of neural networks and back-propagation algorithms in learning. Back-propagation has poor stability in the presence of discontinuity, exhibits high stiffness, and suffers from problems related to local minima, all of which, in practice, restrict the use of neural control in major applications.

Deterministic optimization methods such as Steepest Descent, Conjugate Gradient and Newton's methods are known for their stability in iteratively reducing the objective function value for minimization problems. However, they are primarily suitable for solving single objective function problems that are unimodal and continuous. In comparison, the PSO algorithm permits all particles to have quantum behavior. Consequently, instead of a Newtonian random walk, some sort of "quantum motion" is imposed on the search process.

Newton-type and quasi-Newton methods exhibit superliner convergence in the vicinity of a non-degenerate optimizer. However, these methods require the Hessian or the gradient, respectively, in contrast to other optimization procedures, like the Nonlinear Simplex method, the direction set method of Powell, or some other recently proposed methods. The developed control strategy ensures that the tele-operated machine behaves like a mechanical tool with full haptic feedback to the operator to feels the forces in real-time with less delay. Under sufficiently low manipulation bandwidth, coordination of the PHANTOM and the slave actuator is achieved. The efficiency of the control scheme has been experimentally validated. Compared to other control schemes, by ensuring the coupling stability of the hydraulic tele-operator with a broad class of objects can be achieved.

\section{References}

[1] Zulfatman \& Rahmat M F (2009) Application of self-tuning fuzzy PID controller on industrial hydraulic actuator using system identification approach. International journal on smart sensing and intelligent systems Vol. 2 No. 2

[2] Tandon B, Kaur R (2011) Genetic algorithm based parameter tuning of PID controller for composition control system. International Journal of Engineering Science and Technology (IJEST) ISSN: 09755462 Vol. 3 No. 8 page: $6705-6711$

[3] Kim J S, Kim J H, Park J M, Park S M, Choe W Y and Heo H (2008) Auto tuning PID controller based on improved genetic algorithm for reverse osmosis plant, World Academy of Science, Engineering and Technology 47

[4] Kim D H, Cho J H (2004) Robust tuning for disturbance rejection of PID controller using evolutionary algorithm. North American Fuzzy Information Processing Society Conference (NAFIPS) (1) 248-253

[5] Panduro M A, Brizuela C A, Balderas L I and Acosta D A (2009) A comparison of genetic algorithms, particle swarm optimization and the differential evolution method for the design of scannable circular antenna arrays. Progress in Electromagnetic Research B Vol. 13 171186

[6] Hagan M, Demuth H (1999) Neural Networks for Control. Invited Tutorial, 1999 American Control Conference pp 1642-1656

[7] Hirvonen M, Pyrhönen O and Handroos H (2006) Adaptive nonlinear velocity controller for a flexible mechanism of a linear motor. Mechatronics, 16(5), 279-290

[8] Byrne J P (2003) Genetic Algorithm - Optimization of a Fuzzy Logic Controller, School of Electronic Engineering, Dublin City University, Date: August 2003
[9] Oliveira G T and Saramago S F (2007) A contribution to the study about differential evolution, Ciência \& Engenharia, v. 16, n. 1/2, p. 1 - 8, jan. - dez. 2007

[10] Singiresu S (1996) Engineering Optimization: Theory and Practice, 3rd ed., John Wiley \& Sons, Inc.

[11] Carlisle A and Dozier G (2001) An Off-The-Shelf PSO, In Proceedings of the PSO Workshop, pp 1-6, 2001

[12] El-Telbany M E (2007) Employing Particle Swarm Optimizer and Genetic Algorithms for Optimal Tuning of PID Controllers: A Comparative Study. ICGST International Journal on Automatic Control and Systems Engineering (ACSE) Volume 07 Issue II Pages 49-54

[13] Kennedy J and Eberhart R (1995) Particle swarm optimization. IEEE International Conference on Neural Networks - Conference Proceedings, 4 1942-1948

[14] Eberhart R C and Shi Y (1998) Comparison between genetic algorithms and particle swarm optimization, IEEE Int. Conf. Evol. Comput., Anchorage, pp 611-616

[15] Veeramachaneni K, Peram T, Mohan C and Osadciw L (2003) Optimization Using Particle Swarm with Near Neighbor Interactions, Lecture Notes Computer Science, Springer Verlag

[16] Tandon B, Kaur R (2011) Genetic alghoritm based parameter tuning of PID controller for composition control system, International Journal of Engineering Science and Technology (IJEST), ISSN : 0975-5462 Vol. 3 No. 8 page: 6705-6711

[17] Halme J, Handroos H (1997) Utilization of genetic algorithm in online tuning of power servos, Lappeenranta University of Technology

[18] Shi Y and Eberhart R C (1998) Parameter selection in particle swarm optimization. Indiana University Purdue University; Indianapolis.

[19] Yousefi. H., Hirvonen, M., Handroos, H. and Soleymani, A. 2008. Application of neural network in suppressing mechanical vibration of a permanent magnet linear motor. Control Engineering Practice, 16(7), 787-797

[20] H. Yousefi, H. Handroos and J. K. Mattila (2007). Application of fuzzy gain-scheduling in position control of a servo hydraulic system with a flexible load. International Journal of Fluid Power, 8(2), 2535. Retrieved from www.scopus.com

[21] H. Yousefi (2007) On modeling system identification and control of servo-Systems with a flexible load, (Doctoral Thesis, Lappeenranta University of Technology), ISBN 978-952-214-397-6, ISSN 14564491

[22] Application of Neural Network in Suppressing Mechanical Vibrations of a Permanent Magnet Linear Motor, Yousefi, H., Hirvonen, M., Handroos, H., , Control Engineering Practice, (In Press)

[23] Canudas de Wit C, Olsson H, Åström K J, Lischinsky P (1995) A new model for control of systems with friction. IEEE Transactions on Automatic Control 40(3):419-425

[24] Olsson H. Control systems with friction. PhD thesis. Lund Institute of Technology 1996; University of Lund, Sweden.

[25] Liu J, Handroos H, Haario H, Wu H (2009) Reliability analysis of a model for servo hydraulic system by utilizing MCMC. 7th Int'l Conf. on Fluid Power and Control China

[26] Pap R. What are the advantages of MCMC based inference in latent variable models? Statistica Neerlandica 2002; Volume 56, No.: 1, pages: 2-22, Published by Blackwell.

[27] Asai M. Comparison of MCMC methods for estimating Garch models. J. Japan Statist. Soc 2006; Vol. 36 No. 2 199-212.

[28] Eberhart R.C. and Shi Y., "Comparing Inertia Weights and Constriction Factors in Particle Swarm Optimization", Proceedings of the Congress on Evolutionary Computation, pp 84-88, 2000 - 160

[29] Shi Y. and Eberhart R.C., "A modified particle swarm optimizer", Proceedings of the IEEE International Conference on Evolutionary Computation, pp 69-73, 1998

[30] Abbas A, Haider S (2009) Comparison of AIS and PSO for constrained portfolio optimization. International Conference on Information and Financial Engineering(ICIFE) 50-54

[31] S. M. Giriraj Kumar, D. Jayaraj and A. R. Kishan (2010) PSO based tuning of a PID controller for a high performance drilling machine, International Journal of Computer Applications (0975 - 8887), Volume 1 - No. 19

[32] Carlisle A. and Dozier G., "An Off-The-Shelf PSO", In Proceedings of the PSO Workshop, pp 1-6, 2001

[33] Korani, W.M.; Dorrah, H.T.; Emara, H.M, Bacterial foraging oriented by Particle Swarm Optimization strategy for PID tuning, 
Cairo University, page(s): 445 - 450, Daejeon, ISBN: 978-1-42444808-1, 15-18 Dec. 2009.

[34] Korani, W. M., Dorrah, H. T. and Emara, H. M. 2009. Bacterial foraging oriented by particle swarm optimization strategy for PID tuning. Proceedings of IEEE International Symposium on Computational Intelligence in Robotics and Automation, CIRA, 445450

[35] Goldberg D.E., "GA in search, optimization and machine learning", Addison- Wesley, 1989

[36] Dorigo M., Gambardella L.M., "Ant Colony System: A Cooperative Learning Approach to the Traveling Salesman Problem", IEEE Transactions on Evolutionary Computation, pp.53-66, 1997

[37] Bonabeau E., Dorigo M. and Theraulaz T., "From Natural to Artificial Swarm Intelligence", Oxford University Press, 1999

[38] SensAble Technologies Inc. 2004. PHANTOM PREMIUM, User's Guide, Part \# 02321, Revision 2.0

[39] André Gsponer and Jean-Pierre Hurni, "ITER: The International Thermonuclear Experimental Reactor and the Nuclear Weapons Proliferation Implications of Thermonuclear-Fusion Energy Systems", Independent Scientific Research Institute, Box 30, CH1211 Geneva-12, Switzerland, e-mail: isri@vtx.ch, ISRI-98-01.30 ISRI-04-01.12 January 23, 2004

[40] ITER Documentation Series NO. 22, International Thermonuclear Experimental Reactor (ITER), Engineering Design Activities (EDA) Summary of ITER Final Design Report, July 2001, International Atomic Energy Agency, Vienna, 2001 